\title{
Self-Confirming Price-Prediction Strategies for Simultaneous One-Shot Auctions*
}

\author{
Michael P. Wellman ${ }^{1}$, Eric Sodomka ${ }^{2}$, and Amy Greenwald ${ }^{2}$ \\ ${ }^{1}$ Computer Science \& Engineering, University of Michigan \\ ${ }^{2}$ Computer Science, Brown University
}

December 23, 2016

\begin{abstract}
Bidding in simultaneous auctions is challenging because an agent's value for a good in one auction may depend on the outcome of other auctions; that is, bidders face an exposure problem. Given the gap in our understanding of (e.g., lack of game-theoretic solutions to) general simultaneous auction games, previous works have tackled the problem of how to bid in these games with heuristic strategies that employ probabilistic price predictions-so-called price-prediction strategies. We introduce a concept of self-confirming prices, and show that within an independent private value model, Bayes-Nash equilibrium can be fully characterized as a profile of optimal price-prediction strategies with self-confirming prices. We operationalize this observation by exhibiting a practical procedure to compute near-selfconfirming price predictions given a price-prediction strategy. An extensive empirical game-theoretic analysis demonstrates that bidding strategies that use such predictions are effective in simultaneous auctions with both complementary and substitutable preference structures. In particular, we produce one such strategy that finds near-optimal bids, thereby outperforming all previously studied bidding heuristics in these environments.
\end{abstract}

\section{Introduction}

One of the most promising features of automated trading is the ability to monitor prices and trade in many markets simultaneously. Compared to human traders, automated traders can take in data from many more sources at much higher throughput rates. In principle, automated traders can also process massive quantities of information relevant to trading decisions in short time spans. In practice, however, dealing with multiple markets poses one of the greatest strategic challenges for automated trading. When markets interact, a strategy for trading in one market must consider the ramifications on others.

\subsection{Problem Addressed}

Markets are interdependent when an agent's preferences over outcomes in one depend on outcomes in others. For instance, an agent's value for one good can increase by obtaining another (such goods are called

\footnotetext{
* Substantially extends a version with the same title appearing in the Proceedings of the Twenty-Eighth Conference on Uncertainty in Artificial Intelligence, August 2012.
} 
complements). Complementary preferences for goods in multiple markets gives rise to the classic exposure problem: before it can obtain a valuable bundle, an agent must risk obtaining only a strict subset of the goods it wants at the prevailing prices. Exposure is a potential issue for substitute goods as well, when the agent risks obtaining a strict superset of the goods it wants at the prevailing prices.

The pitfall of exposure is a primary motivation for combinatorial auctions [Cramton et al., 2005], where the mechanism takes on the responsibility of allocating goods respecting agents' expressed interdependencies. Combinatorial auctions are often infeasible, however, due to nonexistence of an entity with the authority and capability to coordinate markets of independent origin. Consequently, interdependent markets are inevitable. Nonetheless, there is at present very little fundamental understanding of agent bidding strategies for these markets. Specifically, how should an agent address the exposure problem?

We address this question in one very basic form of interdependent markets-simultaneous one-shot sealed-bid (SimOSSB) auctions. Since these markets can be plagued by the exposure problem, they get at the essence of interdependent markets. Yet, despite their simplicity, there is little available guidance in the auction theory literature on the strategic problem of how to bid in these auctions. We aim to fill this gap by providing computationally feasible methods for constructing bidding strategies for SimOSSB auctions, which we justify with both theory and evidence gathered from extensive simulations.

\subsection{Summary of Results}

Our theoretical and experimental findings point to two key ingredients for developing effective bidding strategies for simultaneous one-shot sealed-bid auctions, in the special case of independent private values.

The first is an algorithm for computing approximately optimal bid vectors given predicted market clearing prices. We call the method LocalBid, as it employs a local search approach (§5.4.2), cyclically iterating over the set of goods, on each cycle optimizing the bid for a single good given the current other-good bid vector and the input price prediction. We have found in computational experiments that LocalBid achieves a high fraction of optimality $(\S \mathrm{D})$, and that this translates into superior performance in strategic SimOSSB simulations ( $(6)$.

The second ingredient is an approach to generating price predictions for input to a prediction-based bidding strategy. Specifically, we compute self-confirming price predictions: predictions that give rise to themselves when the given price-prediction bidding strategy is applied to them in a specified auction environment. We exhibit a simple iterative estimation procedure ( $\$ 5.6)$, which we have found to be effective at finding price distributions that are approximately marginally self-confirming for a range of strategies and environments $(\S \mathrm{C})$. We refer to the corresponding bidding strategies as self-confirming price-prediction strategies.

Our theoretical results say that if these ingredients were to accomplish their tasks perfectly (i.e., without any approximation error), they would produce a solution (i.e., a Bayes-Nash equilibrium, BNE) to the corresponding simultaneous auction game $(\S 4)$. This conclusion follows from the fact that for typical single-good sealed-bid auctions, an agent's own bid plus the highest other-agent bid is a sufficient statistic for describing an auction's outcome. Given this fact, the logic behind our BNE characterization is mathematically straightforward, so its significance lies in its generality and that it enables a dramatic reduction in dimensionality for equilibrium reasoning. Specifically, rather than consider best responses to profiles of bidding strategies, it is sufficient to best-respond to distributions over prices. The two ingredients listed above comprise a computational recipe for exploiting this observation in general SimOSSB auction environments.

Of course, perfectly responding to exactly self-confirming price predictions is generally not practical. To somewhat mitigate this concern, we also show that the equilibrium solutions degrade gracefully, in that approximations to the ideal in these ingredients yield approximate game-theoretic solutions $(\S \mathrm{B})$. 
Our computational experiments indicate that following this approach produces results that are as good or better than any other general method proposed for bidding in simultaneous one-shot auctions. The evidence takes the form of a comprehensive simulation-based analysis, covering both complementary and substitutable valuation classes and a broad swath of heuristic strategies from the literature. Systematic simulation of hundreds or thousands of strategy combinations in each setting provides payoff estimates, which we use to construct an empirical normal-form game model. For each game we derive an equilibrium over the heuristic strategies. This analysis demonstrates the efficacy of self-confirming LocalBid compared to all competitors, across five evaluation environments covering qualitatively different valuation classes. A direct comparison of bid-optimization performance also favors LocalBid over other heuristics $(\S \mathrm{D})$.

Overall, the investigation provides strong support for self-confirming price-prediction strategies as a general approach to bidding in SimOSSB games, and establishes LocalBid as a leading computationally feasible bidding strategy.

\section{Related Work}

Theoretical results about bidding in general simultaneous auction games are few and far between. The leading auction theory textbook [Krishna, 2010] treats sequential but not simultaneous auctions, ${ }^{1}$ and the most influential comprehensive survey [Klemperer, 2004] addresses simultaneity only in the context of ascending or multi-unit auctions. Cai and Papadimitriou [2014] recently showed that finding Bayes-Nash equilibria for simultaneous second-price auctions is PP-hard and approximating them is NP-hard, even for configurations where one bidder has monotone submodular valuations and all the rest are additive.

In the first work to derive an equilibrium of a simultaneous-auction game, Engelbrecht-Wiggans and Weber [1979] tackle an example with perfect substitutes, where each agent is restricted to bid on at most two items. Their analysis was performed in the large-limit of auctions and agents, and exhibited a mixed equilibrium where the agents diversify their bids even though the items are indistinguishable. Most of the remaining published theoretical results on SimOSSB auctions are due to Rosenthal and colleagues. Krishna and Rosenthal [1996] studied a second-price setup assuming independent private values with two categories of bidders: local bidders who have value only for a single item, and global bidders who have superadditive values for multiple items. The authors characterize an equilibrium that is symmetric with respect to the global bidders, and show, somewhat surprisingly, that an increase in the number of bidders often leads to less aggressive bidding. Rosenthal and Wang [1996] tackled a first-price setup, assuming synergies and common values. Szentes and Rosenthal [2003] studied a class of two-bidder auctions with three identical objects; in their model, bidders' marginal values are complete information and are first increasing but then decreasing in the number of items won.

Recently, Rabinovich et al. [2013] generalized fictitious play to games of incomplete information with finite actions, and applied their technique to a class of simultaneous second-price auctions. The authors compute approximate equilibria for environments with complements and substitutes where utilities can be expressed as linear functions over a one-dimensional type space.

There has also been much recent theoretical attention devoted to bounding the price of anarchy in SimOSSB auctions: the ratio between the expected ex-post optimal allocation and the worst-case expected allocation value among equilibrium solutions. Christodoulou et al. [2008] showed that for simultaneous second-price auctions with submodular valuations, the price of anarchy is two: equilibria are no worse than half optimal. For first-price auctions with submodular valuations, the ratio is at most $\frac{e}{e-1} \approx 1.58$

\footnotetext{
${ }^{1}$ This despite the fact that its author is responsible for some of the few results [Krishna and Rosenthal, 1996] in this area.
} 
[Syrgkanis, 2012]. Feldman et al. [2013] considered the more general class of complement-free (subadditive) valuations, and established bounds of two for first-price and four for second-price. All of these results forbid bidding above value; for this reason, analyses along these lines are unlikely to produce useful bounds for complementary valuations [Feldman et al., 2013].

Hassidim et al. [2011] study price of anarchy and equilibrium for simultaneous first-price auctions under complete information. They find that when pure equilibria exist they correspond to efficient Walrasian price equilibria, and that mixed equilibria (which they also term non-price equilibria) generally exist for some tie-breaking rule.

In the absence of analytic solutions, researchers designing trading strategies for simultaneous auctions (with both complements and substitutes) have addressed the exposure problem through heuristic means. For example, in the Trading Agent Competition (TAC) Travel game [Wellman et al., 2007], agents face an exposure problem for hotels - they must obtain a room for each night for the client, otherwise the whole trip is infeasible. Experience from TAC and many other domains has demonstrated the importance of price prediction for bidding in interdependent markets [Wellman et al., 2004]. Given probabilistic predictions of prices across markets, the agents attempt to manage exposure risk, choosing bids that trade off the profits and losses of the possible bundles of goods they stand to win.

Greenwald and Boyan [2004] framed the problem of bidding across interdependent markets given probabilistic price predictions. Follow-on work [Greenwald et al., 2009, Wellman et al., 2007] formalized this bidding problem in decision-theoretic terms, and established properties of optimal bidding strategies given the assumption that bids do not affect other-agent behaviors. Further experimental comparison was performed by Greenwald et al. [2010]. These works introduced a taxonomy of heuristic bidding strategies [Wellman, 2011], which we employ in the current study.

Self-confirming price-prediction bidding strategies [MacKie-Mason et al., 2004] were first explored in the context of simultaneous ascending auctions (SimAAs) [Cramton, 2005]. For the SimAA environment, these strategies were found to be highly effective at tackling the exposure problem [Wellman et al., 2008].

\section{Model}

We consider a market with $m$ goods, $\mathcal{X}=\{1, \ldots, m\}$, and $n$ agents. The $m$ goods are allocated to the agents via SimOSSB auctions, one per good. That the mechanism is simultaneous means that each agent $i$ submits a bid vector $\boldsymbol{b}_{i}=\left\langle b_{i}^{1}, \ldots, b_{i}^{m}\right\rangle \in \mathbb{R}_{+}^{m}$ before a specified closing hour. That the auctions are oneshot means that, upon the closing, the outcome (who wins each good, and what each agent's payment is) is computed and announced. That the bids are sealed means that agents have no information about the bids of other auction participants until the outcome is revealed.

The other defining characteristic of SimOSSB auctions is that outcomes are determined independently. That is, the allocation of good $j$ and the amount that its winner pays depends only on the bids for good $j$, not those for other goods. Formally, let $w_{i}^{j}\left(b_{i}^{j}, b_{-i}^{j}\right) \in[0,1]$ denote the probability that agent $i$ wins good $j$, given that the other agents bid $b_{-i}^{j}=\left\langle b_{1}^{j}, \ldots, b_{i-1}^{j}, b_{i+1}^{j}, \ldots, b_{n}^{j}\right\rangle$. Similarly, $\psi_{i}^{j}\left(b_{i}^{j}, b_{-i}^{j}\right) \in \mathbb{R}$ denotes the payment assessed to agent $i$, conditional on winning good $j$, given these bids.

Definition 1 (Anonymity). The auction for good $j$ is anonymous if the functions $w_{i}^{j}$, for $i \in\{1, \ldots, n\}$, are identical, and likewise for $\psi_{i}^{j}$.

We assume henceforth that the auctions are anonymous, and accordingly drop the agent subscripts on $w$ and $\psi$. 
Agent $i$ 's value for a bundle $X \in 2^{\mathcal{X}}$ is given by $v_{i}(X)$, where $v_{i}(X) \in[0, \bar{V}]$. We assume free disposal: if $X \subseteq X^{\prime}$, then $v_{i}(X) \leq v_{i}\left(X^{\prime}\right)$. Agent $i$ 's utility $u_{i}$ for the auction outcome is its value for the bundle of goods won net of payments. Our investigation employs the familiar independent private values (IPV) model (see, for example, Krishna [2010]), which means that agents' valuation functions $v_{i}$ are drawn independently from commonly known probability distributions. Under IPV, knowing other agents' valuations tells an agent nothing about its own value, so the fact that the other-agent bids are induced by their valuations is irrelevant, conditional on the bids themselves [Wellman et al., 2011]. Agent $i$ 's utility under IPV can thus be written as follows:

$$
u_{i}\left(\boldsymbol{b}_{i}, \boldsymbol{b}_{-i}\right)=v_{i}\left(w\left(\boldsymbol{b}_{i}, \boldsymbol{b}_{-i}\right)\right)-\psi\left(\boldsymbol{b}_{i}, \boldsymbol{b}_{-i}\right) .
$$

The second-price sealed-bid (SPSB) auction is an OSSB auction in which the winning bidder pays the second-highest bid rather than its own (highest) bid. The environments studied in our empirical gametheoretic analysis below employ the SPSB mechanism. Our description throughout also focuses on SPSB, although our theoretical results hold as well for first-price sealed-bid (FPSB) auctions, or indeed any auction mechanism satisfying the following condition, which says that the outcome to agent $i$ is a function of $i$ 's own bid and the highest other-agent bid.

Definition 2 (Highest-bid (HB) sufficiency). Let $b_{-i}$ and $b_{-i}^{\prime}$ be two other-agent bid vectors for a singlegood auction, such that $\max _{k \neq i} b_{k}=\max _{k \neq i} b_{k}^{\prime}$. The auction mechanism is $H B$-sufficient if for any such vectors and any $b_{i}, w\left(b_{i}, b_{-i}\right)=w\left(b_{i}, b_{-i}^{\prime}\right)$ and $\psi\left(b_{i}, b_{-i}\right)=\psi\left(b_{i}, b_{-i}^{\prime}\right)$.

Strictly speaking, FPSB and SPSB auctions are not HB-sufficient, due to the possibility of ties among varying numbers of bidders. For example, assuming that ties are broken uniformly at random, the probability of winning given a tie depends on how many other agents are in the tie, which cannot generally be determined from the highest other-agent bid alone. However, as we argue in $\S 4$, under a modest assumption, the prospect of ties with any consequence is negligible in our setting.

Ignoring ties so that we can assume HB-sufficiency, we further simplify notation and let $w(\boldsymbol{b}, \boldsymbol{q})=\{j \mid$ $\left.b^{j}>q_{j}\right\}$ denote the set of goods an agent would win by bidding $\boldsymbol{b} \in \mathbb{R}_{+}^{m}$ when the highest other-agent bids are $\boldsymbol{q} \in \mathbb{R}_{+}^{m}$, and $\psi(\boldsymbol{b}, \boldsymbol{q})$ the total price it pays for goods it wins. For example, the payment rule for first-price auctions is $\psi(\boldsymbol{b}, \boldsymbol{q})=\sum_{j \in w(\boldsymbol{b}, \boldsymbol{q})} b^{j}$, and for second-price auctions $\psi(\boldsymbol{b}, \boldsymbol{q})=\sum_{j \in w(\boldsymbol{b}, \boldsymbol{q})} q_{j}$. Given HB-sufficiency, agent $i$ 's utility for a bid depends only on highest other-agent bids, so (1) can be rewritten:

$$
u_{i}(\boldsymbol{b}, \boldsymbol{q})=v_{i}(w(\boldsymbol{b}, \boldsymbol{q}))-\psi(\boldsymbol{b}, \boldsymbol{q}) .
$$

\section{Price-Prediction Strategies and Equilibrium}

For a single SPSB auction with IPV, it is a dominant strategy for an agent to bid its true value. This result does not generalize to simultaneous SPSB auctions, however, unless the agent's value for a bundle of goods happens to be additive. When agents' values for bundles interdepend (e.g., through complementarity or substitutability), bidding truthfully in simultaneous auctions is not even an option, as the value for an individual good is not necessarily well-defined.

To deal effectively with interdependent markets, an agent's bid in each auction must reflect its beliefs about the outcomes of others. We consider beliefs in the form of predictions about the prices at which the agent might obtain goods in the respective auctions. Bidding strategies that are explicitly cast as functions of some input price prediction are termed price-prediction $(P P)$ strategies.

We denote by $p_{j} \in \mathbb{R}_{+}$a price for good $j$. The vector $\boldsymbol{p}=\left\langle p_{1}, \ldots, p_{m}\right\rangle \in \mathbb{R}_{+}^{m}$ associates a price with each good. We represent a price prediction by a probability distribution over the joint price space. We use 
the symbol $\Pi$ to denote the cumulative probability distribution, as follows:

$$
\Pi_{\boldsymbol{p}}(\boldsymbol{q})=\operatorname{Pr}(\boldsymbol{p} \leq \boldsymbol{q}),
$$

where $\boldsymbol{p} \leq \boldsymbol{q}$ holds iff $p_{j} \leq q_{j}$ for all $j$. We generally omit the subscript $\boldsymbol{p}$ as understood.

Definition 3 (PP Strategies). A PP bidding strategy $s(\Pi)$ maps a price prediction to a probability distribution over bid vectors. The expression $s(\Pi)(\boldsymbol{b})$ denotes the probability that strategy $s$ bids $\boldsymbol{b}$ given prediction $\Pi$.

We have elsewhere argued [Wellman, 2011, Wellman et al., 2007] that price prediction is a key element of trading agent architecture, for a broad range of complex trading environments. Here we make a stronger claim: given IPV and HB-sufficiency, PP strategies are necessary and sufficient for optimal bidding in SimOSSB auctions. The remainder of this section presents our support for this claim.

Definition 4 (Optimal PP Bidders). An optimal PP bidding strategy $s^{*}(\Pi)$ submits bids that maximize expected utility given a price prediction $\Pi$ :

$$
s^{*}(\Pi)\left(\boldsymbol{b}^{*}\right)>0 \Longrightarrow \boldsymbol{b}^{*} \in \arg \max _{\boldsymbol{b}} \mathbb{E}_{\boldsymbol{q} \sim \Pi}\left[u_{i}(\boldsymbol{b}, \boldsymbol{q})\right] .
$$

where $u_{i}$ is as defined by (2).

In games of incomplete information like auctions, each agent's strategy produces actions (here, bids) as a function of its type (here, valuation). As argued above, given IPV, utility does not depend on other-agent valuations given their bids. It follows that a best response to any profile of other-agent strategies depends only on the distribution of other-agent bids [Naroditskiy, 2009, Rabinovich et al., 2013].

Furthermore, for HB-sufficient auctions the distribution of highest other-agent bids is a sufficient statistic for the other-agent bid distribution. This highest-bid distribution can be expressed in the form of a price prediction (3). Therefore, a best response to other-agent bidding strategies takes the form of an optimal PP bidding strategy (4), where the price prediction that is input to the PP strategy is the distribution of highest other-agent bids induced by the other-agent strategies.

Lemma 1. A best response to other-agent bidding strategies takes the form of an optimal PP bidding strategy (4), where the price prediction that is input to the strategy is the distribution of highest other-agent bids induced by the other-agent strategies.

Next, we address the issue of auctions that are not strictly HB-sufficient, due to the possibility of ties. In $\S$ A.4 we show by example that an optimal PP bidding strategy is not necessarily a best-response to otheragent bidding strategies when ties are salient. In the setting we investigate empirically, ties are quite unlikely, as bids range over a continuous domain and agent valuations are drawn from a rich space. Furthermore, ties among more than two bids - the necessary condition for HB-sufficiency to fail—are still more unlikely in our setting. Moreover, under an assumption about the interaction between ties and other-agent bid distributions, the loss from playing an optimal PP bidding strategy is negligible, even in settings where ties are common.

Assumption 2. The other-agent strategies are such that the distribution over highest other-agent bids is independent of the number of agents placing the highest bid.

In other words, the fact that there is a tie for highest among some number of other agents does not provide information about the level of this high bid. Although there is no reason to expect any environment to precisely satisfy this assumption, we contend that there is also generally little reason to expect an important dependence between number and level of highest other-agent bids. In order for ties to meaningfully subvert HB-sufficiency, exact matches of more than two agents' bids have to be quite likely, and this dependence would have to be suffienctly strong, and in a particular direction that would be pivotal for a decision. 
Theorem 3. Under Assumption 2, and for any $\tau>0$, the expected utility for optimizing (i.e., playing an optimal PP bidding strategy) with respect to the distribution of highest other-agent bids is within $\tau$ of the expected utility for optimizing with respect to the full distribution of other-agent bids.

The above assumption and theorem are stated more formally as Assumption 7 and Theorem 18 in $\S \mathrm{A}$, which also contains several related proofs. Given Assumption 2, treating SPSB auctions as HB-sufficient entails an infinitesimally small loss in expected utility. Viewed another way, any more significant failure of HB-sufficiency can be traced to a violation of the independence assumption. Henceforth, we adopt Assumption 2 and treat our auctions as essentially HB-sufficient.

Having limited our attention to HB-sufficient auctions, Lemma 1 says that optimal PP bidding strategies constitute best responses to other-agent bidding strategies for the auctions of interest. Since a Bayes-Nash equilibrium (BNE) is nothing more than a profile of mutual best-response strategies, any profile of optimal PP bidding strategies, where the price prediction for each equals the distribution over highest other-agent bids induced by the other agents' (optimal PP) strategies, must constitute a BNE. By the same reasoning, any BNE can be characterized as a profile of optimal PP bidding strategies. We have thus established the following complete characterization of Bayes-Nash equilibria for the SimOSSB auction game.

Theorem 4. Suppose an IPV SimOSSB auction game comprised of HB-sufficient single-good auction mechanisms. The strategy profile $s=\left(s_{1}, \ldots, s_{n}\right)$ is a Bayes-Nash equilibrium if and only if, for all $i, s_{i}$ bids with probability one according to an optimal PP bidding strategy, where agent i's input price prediction is the cumulative distribution of highest other-agent bids: $\Pi_{i}(\boldsymbol{q})=\prod_{k \neq i} \int_{\boldsymbol{p} \leq \boldsymbol{q}} s_{k}\left(\Pi_{k}\right)(\boldsymbol{p}) d p$.

In $\S \mathrm{B}$, we relax the assumption of perfect price prediction, and show how approximate predictions support approximate BNE.

Observe that the price predictions that support Bayes-Nash equilibrium are themselves in a form of equilibrium. Specifically, the bidding strategies employ price predictions that are actually borne out as correct (i.e., the distributions generated by the strategies are as predicted). We refer to this profile of price distributions as self-confirming.

An auction game with IPV is symmetric if all agents have the same probability distribution over valuations. In symmetric games, we can focus on symmetric equilibria, where agents employ a common price prediction and play a common bidding strategy.

Definition 5 (Self-Confirming Price Prediction (SCPP)). Let $\Gamma$ be an instance of a symmetric IPV SimOSSB auction game, and $s$ a bidding strategy that employs price predictions (whether optimally or not). The prediction $\Pi$ is self-confirming for PP strategy $s$ in $\Gamma$ iff $\Pi$ is equal to the distribution of the highest otheragent bids when all agents play $s(\Pi)$.

The next corollary specializes Theorem 4 for the symmetric case, employing the language of SCPPs.

Corollary 5. In symmetric IPV and HB-sufficient SimOSSB auctions, a symmetric BNE comprises optimal $P P$ bidders employing self-confirming price predictions.

Hence, existence of symmetric BNE in such games entails existence of self-confirming price predictions. Indeed, as we find below, the most successful bidding heuristics search for SCPPs.

In summary, for SimOSSB auctions under IPV, we can restrict attention to optimal PP bidding strategies employing price predictions that are self-confirming, meaning they form an equilibrium. ${ }^{2}$ Of course, just because we can does not mean that we should. To make the positive case, in the remainder of this paper, we demonstrate that

\footnotetext{
${ }^{2}$ In the symmetric case, we can focus on a single SCPP distribution.
} 
1. optimal price prediction is amenable to effective approximation;

2. price prediction is a convenient abstraction on which to design and implement a trading strategy; and

3. price prediction strategies exhibit a high degree of robustness across simulation environments.

\section{Heuristic PP Bidding Strategies}

Having shown that optimal PP bidding strategies comprise a BNE, we now turn our attention to practical PP bidding strategies. Given the general infeasibility of exact optimization, ${ }^{3}$ we resort to heuristic methods. Following and building on prior work, we present a broad range of heuristic bidding strategies defined as functions of available price predictions. Our focus in this section is on describing the bidding strategies considered in this study, along with relevant concepts necessary to understand how these strategies operate. Our methods for deriving price predictions are presented in $\S 5.6$.

\subsection{Marginal Values and Optimal Bundles}

Interdependence dictates that the value of any individual good must be assessed relative to a bundle of goods. This idea is captured by the notion of marginal value.

Definition 6 (Marginal Value). Agent $i$ 's marginal value, $\mu_{i}(j, X)$, for good $j$ with respect to a fixed bundle of other goods $X$ is given by $\mu_{i}(j, X) \equiv v_{i}(X \cup\{j\})-v_{i}(X)$.

Given a fixed vector of prices, $\boldsymbol{p}=\left\langle p_{1}, \ldots, p_{m}\right\rangle$, let $\sigma_{i}(X, \boldsymbol{p})$ denote agent $i$ 's surplus from obtaining the set of goods $X$ at those prices: $\sigma_{i}(X, \boldsymbol{p}) \equiv v_{i}(X)-\sum_{j \in X} p_{j}$.

Definition 7 (Acquisition [Boyan and Greenwald, 2001]). Given price vector $\boldsymbol{p}$, the set of acquisitions contains all optimal bundles: $\mathrm{ACQ}_{i}(\boldsymbol{p})=\arg \max _{X \subseteq \mathcal{X}} \sigma_{i}(X, \boldsymbol{p})$.

Faced with perfect point price predictions $\boldsymbol{p}$, an optimal bidding strategy could compute $X^{*} \in \mathrm{ACQ}_{i}(\boldsymbol{p})$ and then bid so as to win precisely those goods in $X^{*}$. By definition, this strategy yields the optimal surplus: $\sigma_{i}^{*}(\boldsymbol{p}) \equiv \sigma_{i}\left(X^{*}, \boldsymbol{p}\right)$.

To assess goods with respect to (typically imperfect) point price predictions, we extend the concept of marginal value. Let $\boldsymbol{p}\left[p_{j} \leftarrow \rho\right]$ be a version of the price vector $\boldsymbol{p}$ with the $j$ th element revised as indicated: $\boldsymbol{p}\left[p_{j} \leftarrow \rho\right]=\left\langle p_{1}, \ldots, p_{j-1}, \rho, p_{j+1}, \ldots, p_{m}\right\rangle$.

Definition 8 (Marginal Value at Prices). Agent $i$ 's marginal value $\mu_{i}(j, \boldsymbol{p})$ for good $j$ with respect to prices $\boldsymbol{p}$ is given by $\mu_{i}(j, \boldsymbol{p})=\sigma_{i}^{*}\left(\boldsymbol{p}\left[p_{j} \leftarrow 0\right]\right)-\sigma_{i}^{*}\left(\boldsymbol{p}\left[p_{j} \leftarrow \infty\right]\right)$.

Here, $\sigma_{i}^{*}\left(\boldsymbol{p}\left[p_{j} \leftarrow 0\right]\right)$ represents agent $i$ 's optimal surplus at the given prices, assuming it receives good $j$ for free. Similarly, $\sigma_{i}^{*}\left(\boldsymbol{p}\left[p_{j} \leftarrow \infty\right]\right)$ represents the optimal surplus at the given prices, if $j$ were unavailable. The difference is precisely the marginal value of good $j$ with respect to its buying opportunities for other goods. Note that Definition 8 generalizes Definition 6, under the interpretation that goods in $X$ have zero price, and all other goods have infinite price.

\footnotetext{
${ }^{3}$ The precise complexity of bid optimization depends on the representations and domains of valuations and price predictions. For some representations, even the problem of bidding given known prices is NP-hard. See $\S 5.5$ for discussion.
} 


\subsection{Bidding with Point Price Predictions}

We consider first a set of PP bidding strategies ${ }^{4}$ that employ predictions in the form of a vector of point prices, $\boldsymbol{p}=\left\langle p_{1}, \ldots, p_{m}\right\rangle$.

The family of strategies TargetBidder restricts bidding to goods in some $X^{*} \in \mathrm{ACQ}_{i}(\boldsymbol{p})$. Members of the family differ in the amounts they bid for these target goods. The TargetPrice strategy bids the predicted price for each good in $X^{*}$.

$$
\text { TargetPrice }_{j}= \begin{cases}p_{j} & \text { if } j \in X^{*} \\ 0 & \text { otherwise }\end{cases}
$$

TargetMV bids at marginal value rather than predicted price.

$$
\operatorname{TargetMV}_{j}= \begin{cases}\mu(j, \boldsymbol{p}) & \text { if } j \in X^{*} \\ 0 & \text { otherwise }\end{cases}
$$

TargetMV* also bids at marginal value, except that it calculates marginal value under the assumption that goods outside the target bundle are unavailable.

$$
\operatorname{TargetMV}_{j}^{*}= \begin{cases}\mu\left(j, \boldsymbol{p}\left[p_{\ell} \leftarrow \infty \mid x_{\ell} \notin X^{*}\right]\right) & \text { if } j \in X^{*}, \\ 0 & \text { otherwise. }\end{cases}
$$

The virtue of the TargetBidder strategies is that they never obtain goods outside their choice bundle. A weakness is their fragility-if the agent fails to acquire all the goods in this bundle, there is no recourse since it has not attempted to acquire any goods outside this set. The StraightMV strategy hedges against incorrect price predictions by bidding marginal value for all goods:

$$
\text { StraightMV }_{j}=\mu(j, \boldsymbol{p}) \text {. }
$$

\subsection{Bidding with Price Distributions}

A point price estimate fails to convey the uncertainty inherent in auction clearing prices. Probability distributions over prices provide a more general representation, expressing degrees of belief over the possible prices that might obtain. We therefore expand our set of heuristics to incorporate bidding methods that take as input a probability distribution over prices. When the input is a distribution rather than point estimates, the bidding problem becomes a stochastic optimization problem.

\subsubsection{Expected Value Methods}

One approach to solving stochastic optimization problems is the expected value method [Birge and Louveaux, 1997], which approximates a solution by collapsing probability distributions into point estimates through expectation. Let $\hat{\boldsymbol{p}}_{\Pi}=\left\langle\hat{p}_{1}, \ldots, \hat{p}_{m}\right\rangle$, where $\hat{p}_{j}=\mathbb{E}_{\boldsymbol{p} \sim \Pi}\left[p_{j}\right]$ is the expected value of $p_{j}$ under price prediction $\Pi$.

Any bidding strategy defined for point price predictions can be adapted to take as input distribution price predictions through this expected value method, simply by using $\hat{\boldsymbol{p}}_{\Pi}$ as the point price prediction. We thus

\footnotetext{
${ }^{4}$ Strategies presented in this section and the next are described with further motivation and detail by Wellman et al. [2007, Chapter 5]. The BidEval meta-strategy ( $\$ 5.4 .1)$ is also discussed there. The LocalBid algorithm ( $\$ 5.4 .2)$ is introduced as a SimOSSB strategy originally for this study.
} 
define StraightMU, TargetMU, and TargetMU* as strategies that take as input distribution predictions, but employ the corresponding point prediction strategies. ${ }^{5}$

In our implementation, we compute the exact expectation $\hat{p}_{\Pi}$ on a piecewise approximation (histogram representation) of $\Pi$. We append the letter "a" (for analytic) to the names of strategies to indicate use of this approach: for example, StraightMUa.

\subsubsection{AverageMU}

Whereas StraightMU bids marginal values at expected prices, $\mu\left(j, \hat{\boldsymbol{p}}_{\Pi}\right)$, an ExpectedMU strategy would bid expected marginal value:

$$
\text { ExpectedMU }_{j}=\mathbb{E}_{\boldsymbol{p} \sim \Pi}[\mu(j, \boldsymbol{p})] .
$$

We approximate this strategy using a heuristic, Average $\mathrm{MU}_{j}$, which samples from the price distribution, calculates marginal values for each sample, and bids the averages of these marginal values across the samples. For example, AverageMU64 draws 64 samples, and then proceeds as described.

\subsection{Explicit Optimization (with Price Distributions)}

Finally, we consider strategies that explicitly attempt to optimize bids given a distribution price prediction, as do the optimal PP bidders (Definition 4).

\subsubsection{BidEval}

One heuristic approach is to first generate candidate bid vectors, and then evaluate them based on the given price distribution $\Pi$. By so doing, the BidEval strategy is actually a meta-heuristic, as it relies on other heuristic bidding strategies to propose candidates. BidEval then predicts each candidate's performance by estimating its expected utility with respect to $\Pi$, and bids as dictated by the candidate with the best estimate.

There are many variations of the BidEval strategy, defined by specifying:

- The method used to generate candidates. When this method is another named bidding strategy, we indicate this fact in parentheses; for instance, BidEval(SMU8) generates candidates using StraightMU8. The strategy BidEvalMix generates candidates using a mix of methods (see $\S \mathrm{E}$ for details).

- Number of candidates generated. A single generation method based on sampling naturally produces a diverse set of candidates. For example, each invocation of StraightMU8 draws a new set of eight samples from $\Pi$, thus we generally obtain different bid vectors.

- Evaluation method. Whether the candidates are evaluated by exact computation on a piecewise approximation of $\Pi$ (again, indicated by an "a" appended to its name), or by sampling. If by sampling, then how many samples are used.

\subsubsection{LocalBid}

The LocalBid strategy (see Algorithm 1) employs a local search method in pursuit of optimal bids. Starting with an initial bid vector (e.g., one proposed by another heuristic), LocalBid makes incremental improvements to that bid vector for a configurable number of iterations. Those improvements are made good-bygood, treating bids for all other goods as constant.

\footnotetext{
5"MU" stands for "marginal utility", but its usage here is simply to distinguish the name from its corresponding point-estimate heuristic ending with "MV".
} 
Fixing bids for all goods except $j$, the agent effectively faces a single auction for good $j$, with the winnings for all other goods determined probabilistically. Rewriting the bid vector as $\boldsymbol{b}=\left(b^{j}, b^{-j}\right)$, an optimal bid $b^{j *}$ for auction $j$ is defined by:

$$
b^{j *} \in \arg \max _{b^{j}} \mathbb{E}_{\boldsymbol{p} \sim \Pi}\left[u_{i}\left(\left(b^{j}, b^{-j}\right), \boldsymbol{p}\right)\right] .
$$

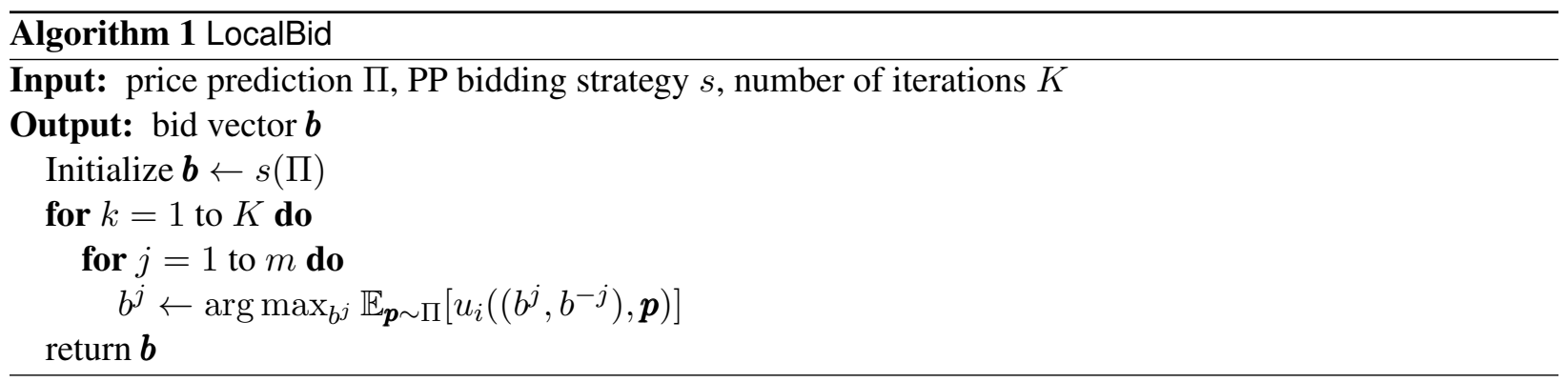

LocalBid is an iterative improvement algorithm: by construction, the expected utility of $\boldsymbol{b}$ is nondecreasing with each update. Further, if LocalBid converges, it returns a bid vector that is consistent in the sense that each entry in the vector is an optimal bid for its corresponding good given the rest of the bid vector. That is, for all goods $j$,

$$
b^{j *} \in \arg \max _{b^{j}} \mathbb{E}_{\boldsymbol{p} \sim \Pi}\left[u_{i}\left(\left(b^{j}, b^{*-j}\right), \boldsymbol{p}\right)\right] .
$$

In the special case of SPSB auctions, under an independence assumption, we can provide a closed form for the LocalBid update rule (5). Recall that in a single SPSB auction, it is a dominant strategy to bid one's value. In simultaneous auctions, agent $i$ 's value for good $j$ hinges on the other goods it wins. But if we assume that the probability distribution over other-good winnings is independent of whether or not it wins good $j$ with any particular bid $b^{j}$, then the agent's bidding problem reduces to the single-good case, with $j$ 's marginal value determined by the probabilistic outcome of the other auctions. It follows that the agent's dominant strategy is to bid its expected marginal value for good $j$, which is given by:

$$
b^{j *}=\mathbb{E}_{\boldsymbol{p} \sim \Pi}[v(w(\boldsymbol{b}, \boldsymbol{p}) \cup\{j\})-v(w(\boldsymbol{b}, \boldsymbol{p}) \backslash\{j\})] .
$$

This expectation can be computed exactly, albeit in time exponential in the number of goods, by iterating over all bundles, calculating the corresponding marginal values, and then weighing these values by the appropriate probabilities (which are easy to look up using our histogram representation of $\Pi$, especially assuming indepdendence).

\subsubsection{OPT}

OPT exhaustively searches the vectors of integer bids, and then iteratively improves the best result found by feeding it to LocalBid. For small discretization factors or a large number of goods, this brute-force heuristic is not computationally feasible. It is therefore not part of our empirical game-theoretic analysis ( $\S 6)$, though we do use it as a benchmark when evaluating the degree of optimality of other heuristics $(\S \mathrm{D})$.

\subsection{Computational Complexity}

We characterize worst-case complexity for the general classes of heuristics implemented here, as a function of the number of goods $m$, the maximum value $\bar{V}$, and algorithmic parameters. For sampling-based heuristics, we denote the number of samples by $N$, and for those employing predictions represented as histograms, 
we assume prices are discretized by integers into buckets from 0 to $\bar{V} \in \mathbb{Z}_{+}$. We further assume that the value function $v$ is explicitly available, so that looking up a bundle's value takes constant time.

The concept of marginal value at prices (Definition 8) is sufficient to characterize optimal acquisitions (Definition 7) [Wellman et al., 2007, Theorem 3.2]. Since optimal acquisition is NP-hard, all known algorithms for computing MV-based heuristics ( $(5.2)$ are exponential in the number of goods, $m$. The expected value variants of these ( $(5.3 .1)$ add to this the cost of computing mean prices, in our implementation totaling $O\left(2^{m}+m N\right)$ for the sample-based methods, or $O\left(2^{m}+m \bar{V}\right)$ for exact calculation from histograms. Our AverageMU heuristic calculates marginal value at each sample, for a total complexity of $O\left(N 2^{m}\right)$.

The BidEval meta-heuristic incurs the cost of generating candidates (which is simply the cost incurred by the input bidding strategy), plus the cost of evaluating them. Evaluating a candidate costs $O(m N)$ for our sample-based methods, or $O\left(m 2^{m}\right)$ using histograms, assuming all bundles must be considered. Given an initial bid, the LocalBid algorithm invokes its core bid update rule $K m$ times. Each update (6) requires essentially the same amount of time as evaluating a bid candidate. Thus, the total complexity of LocalBid given an initial candidate is $O\left(K \mathrm{~m}^{2} \mathrm{~N}\right)$ for sample-based methods, or $O\left(\mathrm{Km}^{2} 2^{m}\right)$ using histograms.

As described above, the computational cost of bidding heuristics can vary greatly depending on the form of valuations, representation of price predictions, and amount of sampling and iteration employed. To ensure feasibility and meaningful comparisons in our experimental analysis, we avoided heuristics and settings that would impose undue computational burdens. At the same time, to ensure generality we refrained from taking computational advantage of any structure in valuation functions. Nevertheless, all of the heuristics implemented computed bids in less than twelve milliseconds on average, on commodity single-core processors, for problems with six goods and significant complementarity in preference. Although we are able to compute bids quite rapidly using any of these heuristics for $m \leq 6$, the exponential worst-case complexity means that scaling to a much larger number of goods would require approximating marginal-value calculations or exploiting structure that may be present in valuations for particular environments.

\subsection{Self-Confirming Price Predictions}

Now that we have a suite of PP strategies, we turn to the question of how to generate price predictions. We employ self-confirming price predictions (SCPPs, Definition 5), originally introduced and evaluated in the context of SimAA auctions [Wellman et al., 2008]. Building on the ideas set forth in this prior work, we propose simple iterative methods to approximate SCPPs.

Algorithm 2 presents our procedure for searching for SCPPs. It takes as input a price-prediction strategy PP as well as several parameters (see Table 1) that control the iterative update process. Let $\pi$ represent a price prediction, in the form of either a point vector or a joint probability distribution. At each iteration, we run $G$ instances of the SimOSSB game, with all agents playing $\operatorname{PP}(\pi)$. We then tally the prices resulting from each instance, and summarize the outcome in the form of another price prediction, $\pi^{\prime}$. For point predictions, $\pi^{\prime}$ is a vector of mean prices; for distribution predictions $\pi^{\prime}$ is a histogram of price outcomes.

During iteration $t$, we update the price prediction by

$$
\pi \leftarrow \kappa_{t} \pi^{\prime}+\left(1-\kappa_{t}\right) \pi
$$

Here, $\kappa_{t}$ is a decaying sequence, which controls the updating of predictions from one iteration to the next. We employ $\kappa_{t}=\frac{L-t+1}{L}$, where $L$ is a limit on the number of iterations. When the distance $\Delta$ between the prediction and observed outcome falls below the threshold $\tau$, the procedure halts and returns $\pi$. For point predictions, we employ

$$
\Delta \equiv \max _{j}\left|\pi_{j}-\pi_{j}^{\prime}\right|
$$




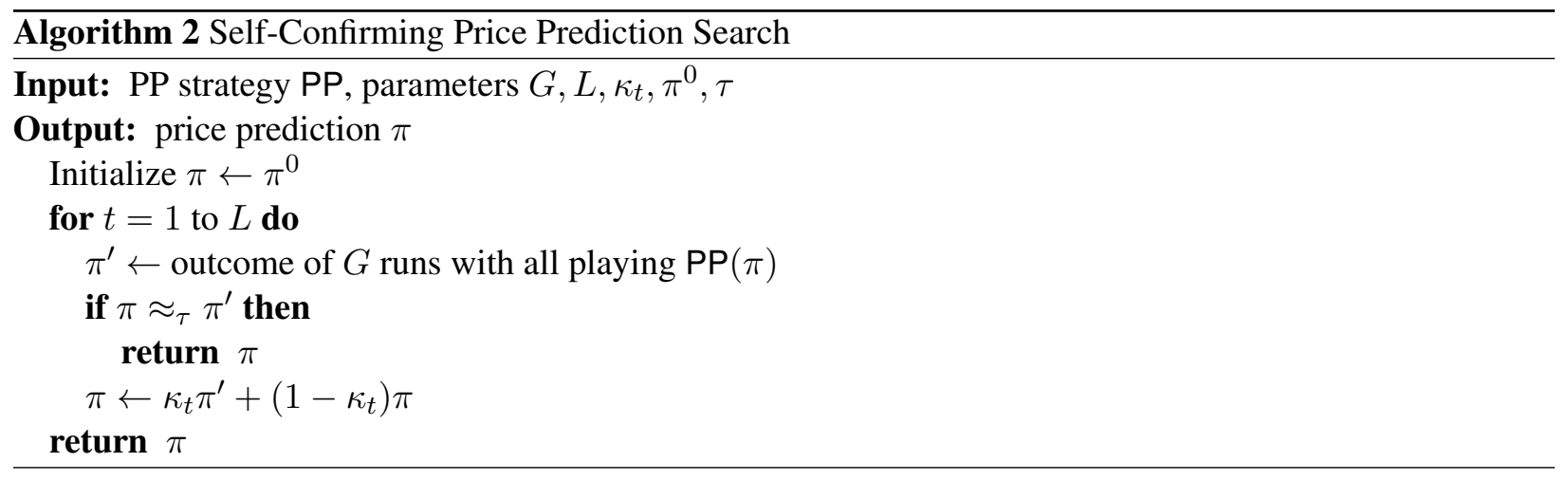

\begin{tabular}{|c|c|c|}
\hline Parameter & Meaning & Value \\
\hline \hline$G$ & number of games per iteration & $10^{5} \leq G \leq 10^{6}$ \\
$L$ & max number of iterations & $100 \leq L \leq 200$ \\
$\kappa_{t}$ & decay schedule & $\frac{L-t+1}{L}$ \\
$\pi^{0}$ & initial prediction (point) & $(1, \ldots, 1)$ \\
$\pi^{0}$ & initial prediction (distribution) & $U[0, \bar{V}]^{m}$ \\
$\tau$ & price distance threshold (point) & $0.001 \bar{V}$ \\
$\tau$ & $K S$ threshold (distribution) & 0.01 \\
\hline
\end{tabular}

Table 1: Parameter settings for self-confirming price prediction procedures.

For distribution predictions, we use the Kolmogorov-Smirnov (KS) statistic. Since we maintain predictions in terms of marginal distributions, our comparison takes the maximum of the KS statistic computed separately for each good:

$$
\Delta \equiv K S_{\text {marg }}\left(\pi, \pi^{\prime}\right) \equiv \max _{j} K S\left(\pi_{j}, \pi_{j}^{\prime}\right) .
$$

If the distance $\Delta$ never falls below threshold, then the procedure terminates after $L$ iterations and returns $\pi$.

The procedure above is open to some flexibility in what one considers as the "outcome" prices resulting from each instance. We explore two versions of the above procedure, one in which this outcome price is the highest other-agent bid (HB), and a second in which it is the actual transaction price of the good (price).

A detailed computational analysis of the convergence and accuracy of the self-confirming price prediction procedure is presented in $\S \mathrm{C}$.

\section{Empirical Game-Theoretic Analysis}

The heuristic strategies introduced in $\S 5$ represent plausible but not generally optimal approaches to bidding in simultaneous auctions. Even the strategies based on explicit optimization ( $(5.4)$ fall short of ideal due to inaccuracy in price prediction and non-exhaustive search of bid candidates. To evaluate the performance of these strategies, we conducted an extensive computational study, simulating thousands of strategy profilesmillions of times each-in five different simultaneous SPSB environments. Analysis of the game model induced from simulation data provides evidence for the efficacy and robustness of approximately optimal PP strategies across these environments. 


\subsection{Approach}

The methodology of applying game-theoretic reasoning to simulation-induced game models is called empirical game-theoretic analysis (EGTA) [Wellman, 2006]. In EGTA, we simulate profiles of an enumerated strategy set playing a game, and estimate a normal-form game from the observed payoffs. The result is a simulation-induced game model, called the empirical game. By then applying standard game-theoretic solution concepts to the empirical game, we can draw conclusions about the strategic properties of the strategies and profiles evaluated. Although the strategy space considered in the empirical game is necessarily a severely restricted subset of the original, by including a broad set of strategies representing leading ideas from the literature, we can produce relevant evidence bearing on the relative quality of heuristic strategies in the simulated environments.

Our EGTA study of simultaneous SPSB environments followed these steps.

1. Define an environment: numbers of goods and agents, and valuation distributions.

2. Specify a set of heuristic strategies. For PP strategies, this includes deriving self-confirming distribution price predictions to be input to these strategies, based on the environment defined in Step 1. The full set of strategies included in our EGTA study is described in $\S \mathrm{E}$.

3. Simulate select profiles among these strategies, sampling from the valuation distributions for each simulation instance (at least one million per profile, most profiles two million or more). Calculate mean payoffs for each strategy in each profile. Scheduling and processing of simulation tasks was substantially automated via our EGTA management infrastructure [Cassell and Wellman, 2013].

4. Analyze the empirical game defined by these mean payoffs, to identify Nash equilibria, dominance relationships, regret values, and other analytic constructs.

In actuality, Steps 2-4 were applied in an iterative and interleaved manner, with intermediate analysis results informing the selection of strategies to explore and profiles to sample. The exploration and sample selection were guided manually, generally driven by the objective of confirming or refuting equilibrium candidates among the profiles already evaluated. A candidate is confirmed when all single-player deviations within the strategy set have been evaluated. The process for each environment was terminated when all of the following conditions were met: (1) a broadly representative set of heuristic strategies were covered, (2) all symmetric mixed profiles evaluated were either confirmed or refuted as equilibria, and (3) all strategies showing relative success in at least one environment were evaluated against the equilibria in all other environments. Overall, the analysis commanded some tens of CPU-years.

\subsection{Environments}

We evaluated five simultaneous SPSB environments, involving 3-8 agents bidding on 5 or 6 goods. The environments span two qualitatively different valuation distributions, one exhibiting strong complements and some substitution, and the other perfect substitutes. These valuations are chosen to exercise a variety of common preference patterns, with a high degree of interdependence among goods. Both of the valuation distributions assume IPV and symmetry, so that each agent receives a private valuation drawn independently from the same distribution. 


\subsubsection{Scheduling Valuations}

The first valuation distribution we employ in this study is based on a model of market-based scheduling [Reeves et al., 2005]. Goods represent time slots of availability for some resource: for example, a machine, a meeting room, a vehicle, or a skilled laborer. Agents have tasks, which require this resource for some duration of time to complete.

Specifically, the goods $\mathcal{X}=\left\{x_{1}, \ldots, x_{m}\right\}$ comprise a set of $m$ time slots available to be scheduled. Agent $i$ 's task requires $\lambda_{i}$ time slots to accomplish, and the agent values a set of time slots according to when they enable completion of the task. If agent $i$ acquires $\lambda_{i}$ time slots by time $t$, it obtains value $v_{i}^{t}$. Value with respect to time is a nonincreasing function: for all $i$, if $t<t^{\prime}$ then $v_{i}^{t} \geq v_{i}^{t^{\prime}}$. If it fails to obtain a sufficient set of goods, the agent accrues value $v_{i}^{\infty}=0$. Let $X \subseteq \mathcal{X}$ denote a set of slots. The expression $\left|\left\{x_{j} \in X \mid j \leq t\right\}\right|$ represents the number of these that are for time $t$ or earlier. We can thus write

$$
T(X, \lambda)=\min \left(\left\{t \text { s.t. }\left|\left\{x_{j} \in X \mid j \leq t\right\}\right| \geq \lambda\right\} \cup\{\infty\}\right)
$$

to denote the earliest time by which $X$ contains at least $\lambda$ slots. The overall valuation function for agent $i$ is then

$$
v_{i}(X)=v_{i}^{T\left(X, \lambda_{i}\right)}
$$

For each agent, a task length $\lambda_{i}$ is drawn uniformly over the integers $\{1, \ldots, m\}$. Values associated with task completion times are drawn uniformly over $\{1, \ldots, 50\}$, then pruned to impose monotonicity [Reeves et al., 2005]. The valuations induced by this scheduling scenario exhibit strong complementarity among goods. When $\lambda>1$, the agent gets no value at all for goods in a bundle of fewer than $\lambda$. On the other hand, there is some degree of substitutability across goods when there may be multiple ways of acquiring a bundle of the required size.

We denote environments using this valuation by $U[m, n]$, with $m$ and $n$ the numbers of goods and agents, and "U" indicating the uniform distribution over task lengths. Our EGTA study covered environments $U[6,4], U[5,5]$, and $U[5,8]$.

\subsubsection{Homogeneous-Good Valuations}

The second valuation distribution expresses the polar opposite of complementarity: goods are perfect substitutes, in that agents cannot distinguish one from another. ${ }^{6}$ Agents' marginal values for units of this good are weakly decreasing. Specifically, valuation is a function of the number of goods obtained, constructed as follows. Agent $i$ 's value for obtaining exactly one good, $v_{i}(\{1\})$, is drawn uniformly over $\{0, \ldots, 127\}$. Its value for obtaining two, $v_{i}(\{1,2\})$, is then drawn from $\left\{v_{i}(\{1\}), v_{i}(\{1\})+1, \ldots, 2 v_{i}(\{1\})\right\}$. In other words, its marginal value for the second good is uniform over $\left\{0, \ldots, v_{i}(\{1\})\right\}$. Subsequent marginal values are similarly constrained not to increase. Its marginal value for the $k$ th good is uniform over $\left\{0, \ldots, v_{i}(\{1, \ldots, k-1\})-v_{i}(\{1, \ldots, k-2\})\right\}$.

We denote environments using this valuation by $H[m, n]$, with " $\mathrm{H}$ " an indicator for homogeneity. Our EGTA study covered environments $H[5,3]$ and $H[5,5]$.

\footnotetext{
${ }^{6}$ Of course a mechanism designer capable of recognizing and addressing this fact would implement a multiunit auction rather than SimOSSB auctions for this case. The case of homogeneous goods allocated in separate auctions actually represents an especially challenging problem for bidding strategy, hence our choice to include it as an evaluation environment.
} 


\subsection{Regret}

We evaluate the stability of a strategy profile by measuring regret, the maximal gain a player could achieve by deviating from the profile. Formally, let $\Gamma=\{n, S, u(\cdot)\}$ be a symmetric normal-form game with $n$ players, strategy space $S$ (the same for each player, since the game is symmetric), and payoff function $u: S \times S^{n-1} \rightarrow \mathbb{R}$. The expression $u\left(s_{i}, s_{-i}\right)$ represents the payoff to playing strategy $s_{i}$ in a profile where the other players play strategies $s_{-i} \in S^{n-1}$.

Definition 9 (Regret). The regret $\epsilon(\boldsymbol{s})$ of a strategy profile $\boldsymbol{s}=\left(s_{1}, \ldots, s_{n}\right)$ is given by

$$
\epsilon(\boldsymbol{s})=\max _{i} \max _{s_{i}^{\prime} \in S}\left(u\left(s_{i}^{\prime}, s_{-i}\right)-u\left(s_{i}, s_{-i}\right)\right) .
$$

A Nash equilibrium profile has zero regret, and more generally regret provides a measure of approximation to Nash equilibrium. Using this regret definition, profile $s$ is an $\epsilon(\boldsymbol{s})$-Nash equilibrium.

Regret is a property of profiles. Evaluation of a particular strategy is inherently relative to a context of strategies played by other agents. Jordan et al. [2007] proposed ranking strategies according to their performance when other agents are playing an equilibrium.

Definition 10 (NE regret [Jordan, 2010]). Let $s^{N E}$ be a Nash equilibrium of game $\Gamma$. The regret of strategy $s_{i} \in S$ relative to $s^{N E}, u\left(s_{i}^{N E}, s_{-i}^{N E}\right)-u\left(s_{i}, s_{-i}^{N E}\right)$, is an $N E$ regret of $s_{i}$ in $\Gamma$.

NE regret represents the loss experienced by an agent for deviating to a specified strategy from a Nash equilibrium of a game. The rationale for this measure comes from the judgment that all else equal, Nash equilibria provide a compelling strategic context for evaluating a given strategy. For games with multiple $\mathrm{NE}$, a given strategy may have multiple NE-regret values.

It is also instructive to examine the regret of strategies in self-play, that is, the gain from deviating from a given strategy when all other agents are playing that strategy.

Definition 11 (Symmetric-profile regret). The symmetric-profile regret $\epsilon(s)$ of a strategy $s \in S$ is the regret $\epsilon(\boldsymbol{s})$ of strategy profile $\boldsymbol{s}=(s, \ldots, s)$, in which each agent plays $s$.

Symmetric-profile regret measures a strategy's degree of approximation to symmetric Nash equilibrium: the profile in which all agents play $s$ is a symmetric $\epsilon(s)$-NE.

\subsection{Results}

Table 2 summarizes the extent of simulation coverage of the five SPSB environments investigated. ${ }^{7}$ The empirical games comprise between 1500 and 20,000 profiles, over 29-34 strategies. For the smallest (threeplayer) game, $H[5,3]$, we were able to simulate all strategy profiles. For the rest, evaluated profiles constitute a small fraction (as little as $0.06 \%$ ) of the entire profile space over these strategies. In all cases, we focused simulation on strategies that demonstrated promise in any context.

To prune the strategy space, we eliminate conditionally dominated strategies, defined as strategies $s$ for which deviating to another particular strategy $s^{\prime}$ improves payoff in every profile where $s$ has been evaluated.

\footnotetext{
${ }^{7}$ Full payoff data for the empirical games is archived at https://deepblue.lib.umich.edu/data/concern/ generic_works/db78tc04f. The results we report here subsume those of a preliminary study [Yoon and Wellman, 2011]. That study set the groundwork for the current investigation by developing the infrastructure for simulation and derivation of selfconfirming price predictions, and tuning parameters (e.g., number of evaluating samples) for several of the strategies. However, the preliminary results reflected sparser coverage of relevant profiles and a weaker overall set of strategy candidates.
} 


\begin{tabular}{|c|c|c|c|c|c|}
\hline Environment & \# Strategies & \# Profiles & \% Profiles & \# CUD Strategies & \% CUD Profiles \\
\hline$U[6,4]$ & 34 & 1575 & 2.38 & 9 & 100 \\
$U[5,5]$ & 30 & 8131 & 1.88 & 14 & 49.6 \\
$U[5,8]$ & 29 & 19520 & 0.06 & 29 & 0.06 \\
$H[5,3]$ & 32 & 5984 & 100 & 15 & 100 \\
$H[5,5]$ & 34 & 17160 & 3.42 & 28 & 7.55 \\
\hline
\end{tabular}

Table 2: Strategies and profiles simulated for the environments addressed in our EGTA study, before and after elimination of conditionally dominated strategies.

As shown in Table 2, for some games fewer than half of the strategies are conditionally undominated (CUD), whereas for a couple of others there is little or no pruning. Restricted to CUD strategies, our simulations provide greater coverage. For $U[6,4]$ and $H[5,3]$ we exhaustively evaluated the CUD profiles, and for $U[5,5]$ we cover all triples of CUD strategies. For the remaining two games we cover all CUD pairs.

We search for symmetric mixed-strategy Nash equilibria in each game by running replicator dynamics [Gintis, 2000, Chapter 9] on every maximal complete subgame: subset of strategies for which all profiles are evaluated. Though replicator dynamics is not a complete algorithm, by trying sufficient starting points we ensured finding at least one equilibrium in each subgame. We then confirmed or refuted these subgame equilibria with respect to the full set of heuristic strategies for each game. For refuted candidates, we made sure to simulate all profiles in the subgame formed by extending the candidate's support with its best response. As it happens, our process identified exactly one symmetric NE in each game; we present these in Table 3. Our coverage patterns and systematic refutatation process provides a basis for confidence that we have not missed small-support symmetric equilibria. Although it is impossible to rule out additional equilibria without exhaustively evaluating the profiles, based on our search experience we would be quite surprised to find any among the enumerated strategies.

\begin{tabular}{|c|c|c|c|c|c|c|c|c|}
\hline & 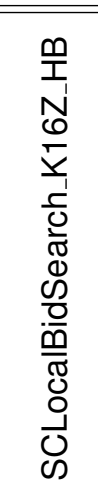 & 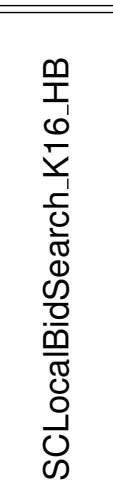 & 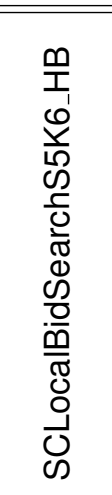 & 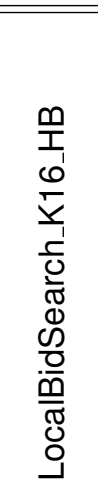 & 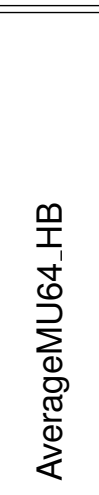 & 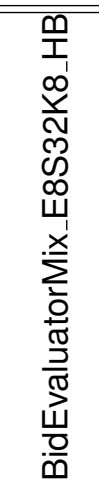 & 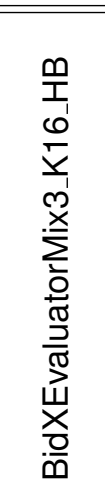 & 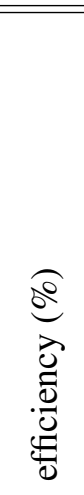 \\
\hline$U[6,4]$ & - & - & - & 0.145 & 0.855 & - & - & 84.8 \\
\hline$U[5,5]$ & - & - & 0.910 & - & 0.090 & - & - & 85.7 \\
\hline$U[5,8]$ & 0.082 & - & 0.918 & - & - & - & - & 84.6 \\
\hline$H[5,3]$ & - & 0.505 & - & 0.409 & - & 0.086 & - & 91.6 \\
\hline$H[5,5]$ & - & 0.635 & - & 0.272 & - & - & 0.093 & 90.5 \\
\hline
\end{tabular}

Table 3: For each environment, probability of each strategy in symmetric mixed equilibrium, as identified through EGTA. The last column records the allocative efficiency of this equilibrium.

Of the 44 distinct strategies explored across environments, only seven were supported in equilibrium in 
any environment. Variants of SCLocalBid, a strategy that explicitly optimizes with respect to self-confirming prices, predominate in equilibrium in four out of five environments. LocalBid and the BidEvalMix strategies also explicitly optimize, but with respect to price predictions that are self-confirming for different strategies (see $\S \mathrm{E}$ ). AverageMU64_HB is the sole non-optimizing heuristic to appear in equilibria, as it performs remarkably well in the complementary-valuation environments.

For each equilibrium found, we measured allocative efficiency: the fraction of optimal allocation value produced on average by the mixture of bidding strategies. The optimal value is computed ex post, that is, as by a central planner who knows all the valuations. As shown in the rightmost column of Table 3 , the equilibrium bidding strategies achieve over $84 \%$ of the available allocation value in the complementarygood environments, and over $90 \%$ in the substitute-good environments. For comparison, the best-known worst-case theoretical bounds are 50\% in the substitutes (submodular) case, and $0 \%$ with complements [Feldman et al., 2013]. Our results provide suggestive evidence that even with significant exposure issues, the price of anarchy need not approach the worst case.

Whether a strategy is in equilibrium or not is a crude binary classification of merit. We measure relative degrees of effectiveness by NE regret (Definition 10), as reported in Figure 1. The figure depicts the NEregret values for 12 top strategies: all those ranked fourth or better in at least one environment. The values are indicated on a horizontal scale for each game environment, ranging from zero (indicating the strategy is in the support of equilibrium) to the highest NE regret value among the listed strategies. We also verified via a bootstrap technique [Wiedenbeck et al., 2014] that the results are statistically robust: for each identified equilibrium the one-tailed $90 \%$ confidence bound on regret is at the far left end (1\% of the range) of the NE regret scale shown.

- SC Local 4 SC BidEval $\square$ Local + BidEval $₫$ AvgMU

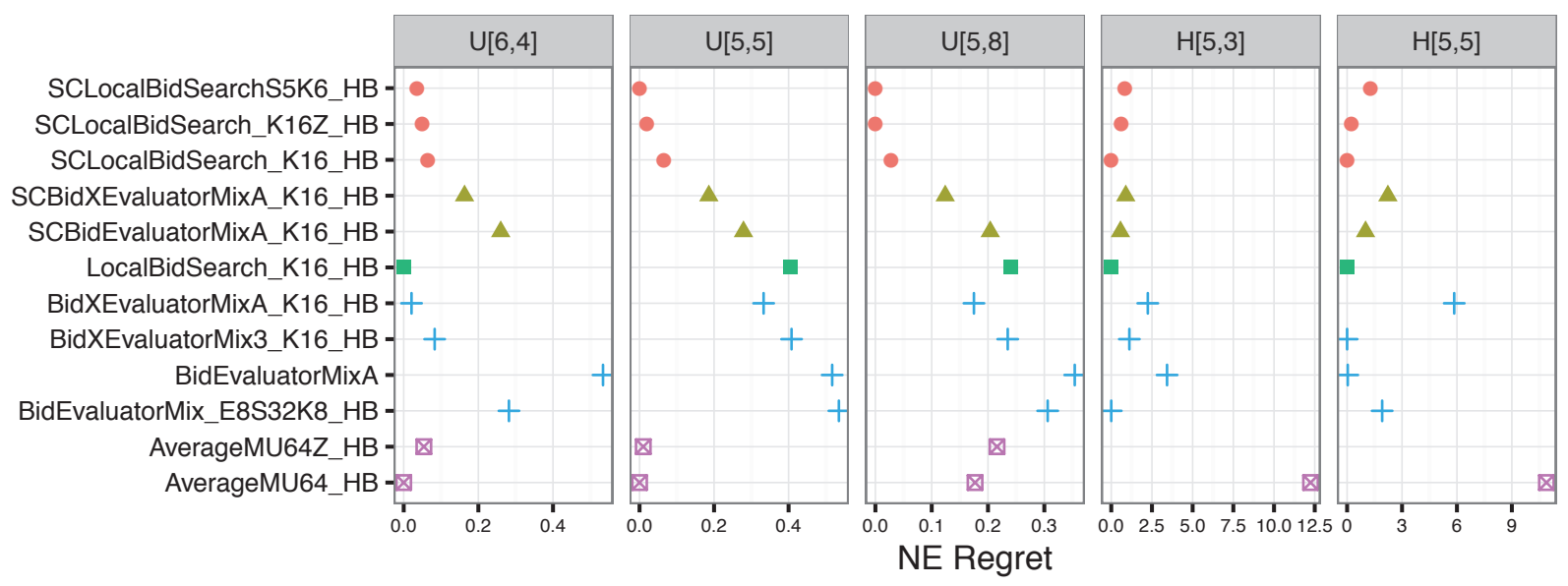

Figure 1: NE regret for top strategies across five environments, measured with respect to the sole equilibrium found in the respective environments.

These results provide solid support for the SCLocalBid strategy. For the one environment it fails to participate in equilibrium, its NE regret is still quite low, thus we find it to be a strong all-around strategy. This situation contrasts starkly with prior findings for bidding in SimAA auctions [Wellman et al., 2008], where the best strategies for complementary (scheduling valuation) environments were awful in substitutes (homogeneous good) environments, and vice versa. The fact that SCLocalBid performs so well aligns with our key theoretical finding, in support of optimal PP bidders with self-confirming price predictions. 
All the remaining top strategies are in the BidEval class (also explicit optimizers), except for AverageMU64. In contrast to the others, however, AverageMU64's observed quality is limited to one of the valuation distributions - the strategy performs poorly in homogeneous-good environments. This observation is consistent with the results reported by Boyan and Greenwald [2001], where an example environment with perfect substitutes was contrived to demonstrate the shortcomings of marginal-utility-based bidding. Given such examples, it is perhaps unsurprising that the heuristic strategies based on marginal value have a difficult time competing with explicit optimizers. If anything it is the observed success of AverageMU64 that is striking, but this outcome is consistent with past experimental results in an environment that exhibits substantial complementarity [Stone et al., 2003]. Still, compared to optimal PP bidders in our experiments, AverageMU lacked robustness across valuation classes.

Similar results are observed for symmetric-profile regret (Definition 11), as shown in Figure 2. The superiority of self-confirming strategies is even more pronounced for symmetric-profile regret than NE regret. This is as expected, since the predictions of self-confirming strategies essentially assume self-play, which is a correct assumption in the symmetric profile.

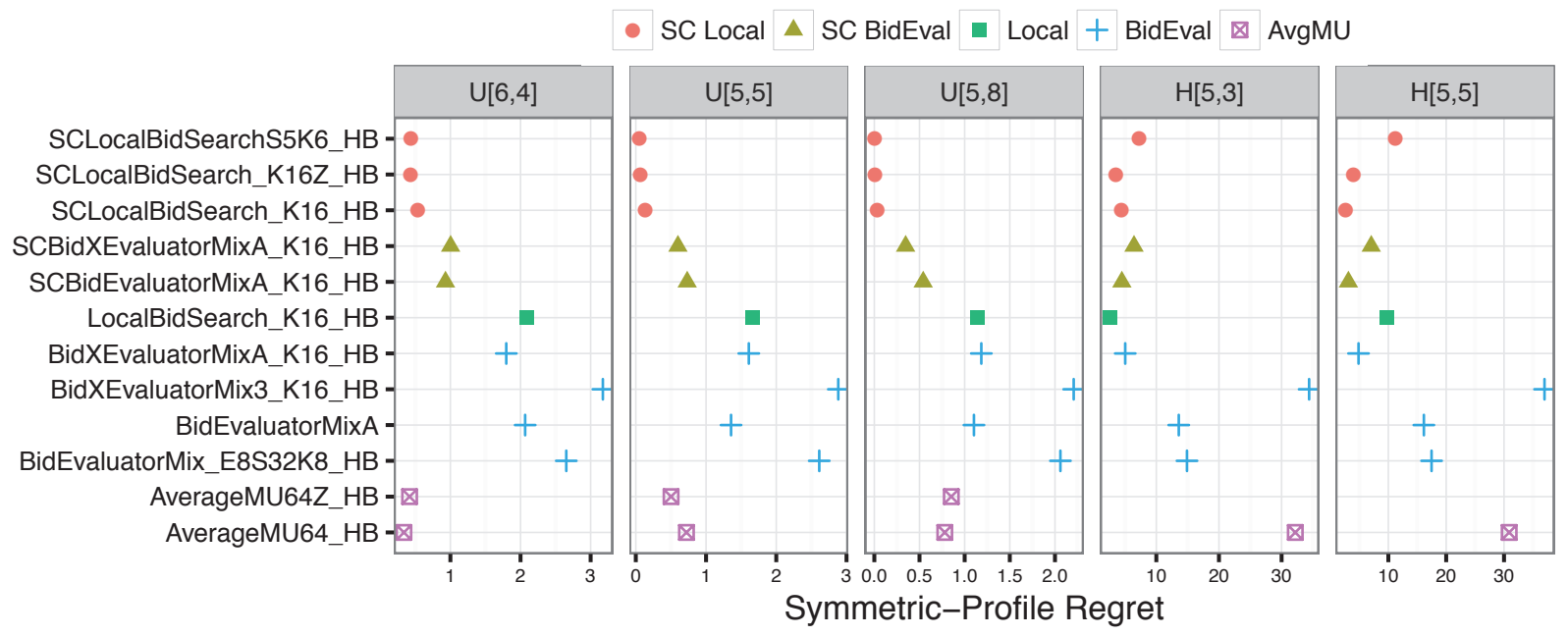

Figure 2: Symmetric-profile regret for top strategies across five environments.

We evaluate the relation between prediction accuracy and game-theoretic stability. Both our theoretical results $(\S 4)$ and empirical accuracy comparisons $(\S \mathrm{C})$ support the use of highest-bid (HB) statistics in deriving self-confirming price distributions, as opposed to the actual transaction price. Indeed, all the top strategies but one use HB predictions. The lone exception in Figure 1 is BidEvaluatorMixA, which performed impressively in one of the homogenous-good environments (despite poor prediction accuracy) but not so well in the rest. Figure 3 shows prediction accuracy (in terms of KS distance) with respect to regret for strategies in the $U[5,5]$ and $H[5,5]$ environments. We see that more accurate predictions tend to have lower NE regret and lower symmetric-profile regret. Overall, of the five pairs of strategies played in $U[5,5]$ that differed only in prediction method, the strategy with more accurate prediction had lower NE regret every time. Of the seven such pairs in $H[5,5]$, the better prediction had lower NE regret in four, and identical NE regret in one. Similar alignment was found for symmetric-profile regret: five out of five in $U[5,5]$, and five out of seven in $H[5,5]$.

Finally, we ran experiments in a decision-theoretic environment to better understand optimization performance of top strategies (see $\S \mathrm{D}$ ). We found that the LocalBid search method was most effective in op- 

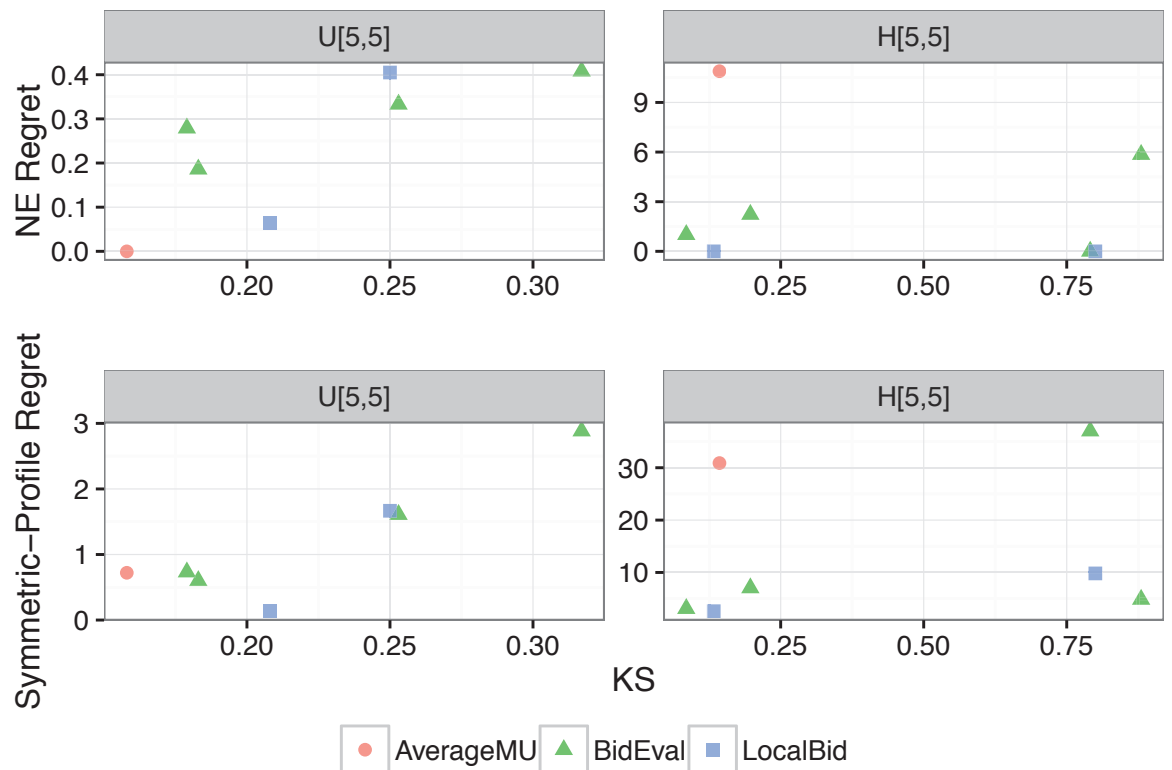

Figure 3: Accuracy of price predictions versus NE regret and symmetric profile regret for top strategies across two environments.

timizing bids at $98.67 \%$ of OPT, followed by BidEval at $94.49 \%$, and lastly AverageMU at $66.10 \%$. To evaluate the relation between optimization performance and game-theoretic stability, we look the average $\mathrm{NE}$ regret and symmetric-profile regret of each strategy class, restricted to the top strategies that appeared in all environments (Figure 4). We see that the strategies that were self-confirming and had better optimization performance had lower average regret across environments. It is consistent with our theory to find that most of the successful strategies were approximately optimal with respect to approximately correct price predictions.
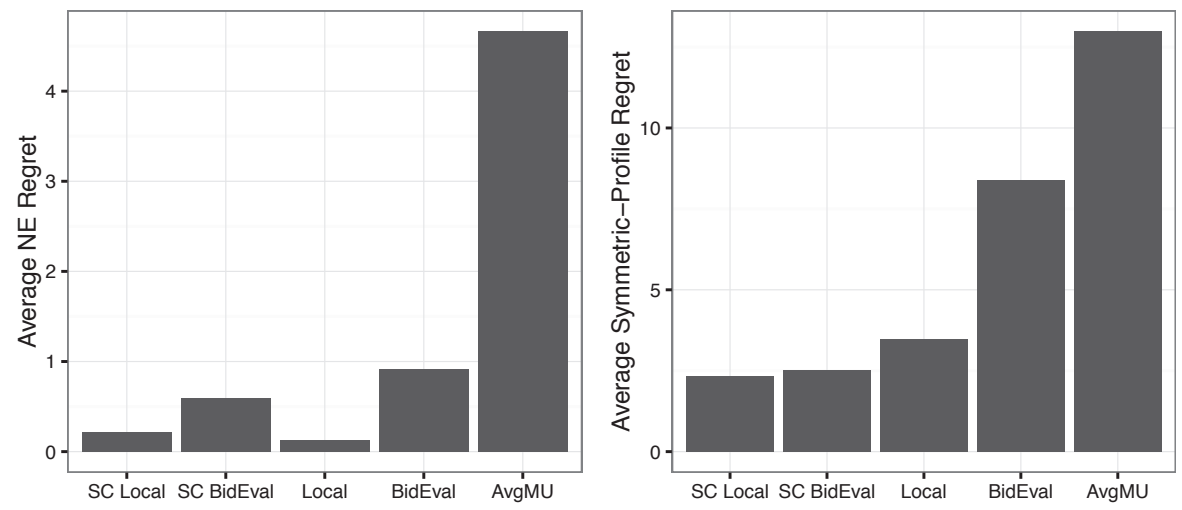

Figure 4: Average (across environments) NE regret and symmetric-profile regret for each strategy class, restricted to the top strategies that appeared in all environments. 


\section{Conclusion}

The main strategic problem in simultaneous auctions is managing the exposure inherent in bidding for some goods without knowing the outcome of auctions for other related goods. Previous research falls in two broad categories: game-theoretic treatment of special environment structures, or evaluation of heuristic methods against fixed opponents. Typically, heuristic strategies deal with exposure by making tradeoffs with respect to probabilistic price predictions.

Our study starts from this concept of price-prediction strategies, and investigates the idea of self-confirming prices, predictions that turn out to be correct when agents optimize with respect to them in bidding. We term bidding algorithms that employ such predictions self-confirming price-prediction (SCPP) strategies. We showed that within an independent private value model, Bayes-Nash equilibrium for simultaneous one-shot sealed-bid (SimOSSB) auctions can be fully characterized as a profile of SCPP strategies. We operationalize this observation through two algorithmic components: (1) a practical procedure to compute near-selfconfirming price predictions given a price-prediction strategy, and (2) heuristic techniques to approximately optimize bids with respect to such predictions. Using an extensive empirical game-theoretic analysis, we demonstrated that heuristic SCPP strategies are effective in simultaneous auctions with both complementary and substitutable preference structures. In particular, we produced one such strategy that finds near-optimal bids, thereby outperforming all previously studied bidding heuristics in these environments.

Having such a practical method for approximating equilibria for SimOSSB auctions can help shed light on the effectiveness of this class of auction mechanisms, as compared to popular alternatives. For instance, our empirical equilibrium for SimSPSB environment $H[5,5]$ is $90.5 \%$ efficient, compared to the $98.5 \%$ efficient equilibrium found for this environment with the SimAA mechanism [Wellman et al., 2008]. In SimAAs with substitutes only, there is no exposure problem [Milgrom, 2000], so the $1.5 \%$ loss of efficiency is due to strategic demand reduction. Thus, we can attribute the additional $8 \%$ loss of efficiency in the SimSPSB setting to exposure. Similar empirical measurements may support comparisons with sequential auctions [Greenwald et al., 2012], complementing comparisons between these mechanisms already provided on a worst-case basis [Paes Leme et al., 2012].

There is still considerable room for improvement in our proposed methods, as well as opportunity to subject our conclusions to further empirical scrutiny. More sophisticated stochastic search techniques may improve upon our best bid optimization algorithms, and allow them to scale to larger environments with more complex valuations. Similarly, we do not consider our simple iterative method to be a last word on finding self-confirming price distributions. One extension we have already begun to explore is the development of price-prediction methods based on joint distributions, which enable bidding heuristics that relax the assumption of probabilistic independence of good prices [Mayer et al., 2013]. As expected, this investigation found an advantage to accounting for price dependencies, at some cost in algorithmic complexity. Interestingly, natural generalizations of the LocalBid method shown superior here also prevailed among methods that exploit joint price predictions.

\section{A Negligibility of Ties}

In this appendix, we analyze more formally the consequences of using PP strategies to bid in SimSPSB auctions. We first introduce additional notation to describe allocation rules, payments, predictions, and utility when auctions are not HB-sufficient. We then provide an overview of all results in this section before delving into the proofs. The key mathematical statements are Assumption 7 and Theorem 18, precise versions of informal counterparts introduced in $\S 4$. Finally, we demonstrate by example that Theorem 18 
does not necessarily hold when Assumption 7 is violated.

\section{A.1 Notation}

Our presentation in $\S 3$ adopted simplified notation for the allocation, payment, and utility functions, based on the presumed HB-sufficiency of the auctions of interest. Here we suspend that presumption, and so must define these functions in terms of all other-agent bids, not just the highest. Expected utility can no longer be computed from the induced distribution over highest other-agent bids $\Pi$, and so we introduce notation for the distribution over all other-agent bids. Additional approximate allocation functions, which are used in subsequent proofs in this section, are also defined.

Throughout, $\boldsymbol{b} \in \mathbb{R}_{+}^{m}$ is a vector of the given agent's bids, $\boldsymbol{q} \in \mathbb{R}_{+}^{m}$ is a vector of highest other-agent bids, and $h \in \mathbb{N}_{+}^{m}$ is a vector giving the number of other agents that placed each highest bid.

Allocation Rules Let $w^{*}$ be the true allocation rule: for each good $j$ in which agent $i$ and $h^{j}$ other bidders have tied for the highest bid, $i$ is allocated good $j$ with probability $1 /\left(h^{j}+1\right)$. We overload notation to express $w^{*}$ in terms of both the probability of winning a particular good $j$ and the probability of winning a bundle of goods $X$ :

$$
\begin{gathered}
w^{*}(j, \boldsymbol{b}, \boldsymbol{q}, \boldsymbol{h})=\left\{\begin{aligned}
1 & \text { if } b^{j}>q^{j} \\
1 /\left(h^{j}+1\right) & \text { if } b^{j}=q^{j} \\
0 & \text { otherwise }
\end{aligned}\right. \\
w^{*}(X, \boldsymbol{b}, \boldsymbol{q}, \boldsymbol{h})=\prod_{j \in X} w^{*}(j, \boldsymbol{b}, \boldsymbol{q}, \boldsymbol{h}) \prod_{j \notin X}\left(1-w^{*}(j, \boldsymbol{b}, \boldsymbol{q}, \boldsymbol{h})\right)
\end{gathered}
$$

Let $\hat{w}_{p}$ be an approximate allocation rule of the following form: if agent $i$ is tied for the highest bid for $\operatorname{good} j$, agent $i$ is allocated good $j$ with probability $p$.

$$
\begin{gathered}
\hat{w}_{p}(j, \boldsymbol{b}, \boldsymbol{q})= \begin{cases}1 & \text { if } b^{j}>q^{j} \\
p & \text { if } b^{j}=q^{j} \\
0 & \text { otherwise }\end{cases} \\
\hat{w}_{p}(X, \boldsymbol{b}, \boldsymbol{q})=\prod_{j \in X} \hat{w}_{p}(j, \boldsymbol{b}, \boldsymbol{q}) \prod_{j \notin X}\left(1-\hat{w}_{p}(j, \boldsymbol{b}, \boldsymbol{q})\right)
\end{gathered}
$$

Let $\widetilde{w}_{Y}$ be an approximate allocation rule with deterministic tiebreakers. If agent $i$ is tied for the highest bid for $\operatorname{good} j$, agent $i$ is allocated $\operatorname{good} j$ with probability 1 if $j \in Y$, and is otherwise allocated $\operatorname{good} j$ with probability 0 .

$$
\begin{gathered}
\widetilde{w}_{Y}(j, \boldsymbol{b}, \boldsymbol{q})= \begin{cases}1 & \text { if } b^{j}>q^{j} \\
1 & \text { if } b^{j}=q^{j} \wedge j \in Y \\
0 & \text { otherwise }\end{cases} \\
\widetilde{w}_{Y}(X, \boldsymbol{b}, \boldsymbol{q})=\prod_{j \in X} \widetilde{w}_{Y}(j, \boldsymbol{b}, \boldsymbol{q}) \prod_{j \notin X}\left(1-\widetilde{w}_{Y}(j, \boldsymbol{b}, \boldsymbol{q})\right)
\end{gathered}
$$

Let $\widetilde{W}=\left\{\widetilde{w}_{Y}\right\}_{Y \subseteq \mathcal{X}}$ be the set of all approximate allocation rules with deterministic tiebreakers. 
Finally, let $w$ be a general allocation rule in which the winner for each good is determined independently, given all bids. The probability of player $i$ winning good $j$ in the instance of a tie is given by $p^{j} .^{8}$

$$
\begin{gathered}
w(j, b, \boldsymbol{q}, \boldsymbol{p})=\left\{\begin{aligned}
1 & \text { if } b^{j}>q^{j} \\
p^{j} & \text { if } b^{j}=q^{j} \\
0 & \text { otherwise }
\end{aligned}\right. \\
w(X, \boldsymbol{b}, \boldsymbol{q}, \boldsymbol{p})=\prod_{j \in X} w(j, \boldsymbol{b}, \boldsymbol{q}, \boldsymbol{p}) \prod_{j \notin X}(1-w(j, \boldsymbol{b}, \boldsymbol{q}, \boldsymbol{p}))
\end{gathered}
$$

When convenient, we use notation that is capable of representing any allocation rule. All inputs to the above allocation rules can be derived from the other-agent bids. Let $w\left(X, \boldsymbol{b}_{i}, \boldsymbol{b}_{-i}\right)$ be the probability that agent $i$ wins bundle $X$ under allocation rule $w$, given $i$ 's bids and all other-agent bids.

Payment Rule In standard auctions, agent $i$ has to pay for good $j$ only when it wins that good. For HB-sufficient auctions, $b_{i}^{j}$ and $q_{i}^{j}$ determine whether $i$ wins good $j$ (as well as $i$ 's payment), but without HB-sufficiency, $i$ may win the good probabilistically. Let $\psi^{j}\left(b_{i}^{j}, b_{-i}^{j}\right)$ be agent $i$ 's payment conditional on winning good $j$. Similarly, let $\psi^{X}\left(\boldsymbol{b}_{i}, \boldsymbol{b}_{-i}\right)$ be agent $i$ 's total payment conditional on winning bundle $X$. For SimSPSB auctions, the payment rule is defined as follows:

$$
\begin{gathered}
\psi^{j}\left(b_{i}^{j}, b_{-i}^{j}\right)=\max _{k \neq i}\left\{b_{k}^{j}\right\} \\
\psi^{X}\left(\boldsymbol{b}_{i}, \boldsymbol{b}_{-i}\right)=\sum_{j \in X} \psi^{j}\left(b_{i}^{j}, b_{-i}^{j}\right)
\end{gathered}
$$

Price Predictions Let $\Psi$ be the induced distribution over other-agent bids, given other-agent strategies and the distribution over other-agent valuations. Formally, let $V_{i}=\mathbb{R}^{\mathcal{X}}$ be the set of possible valuations for agent $i$. Let $f_{i}\left(v_{i}\right)$ give the probability that agent $i$ 's drawn valuation is $v_{i}$. Let $s_{i}: V_{i} \rightarrow \triangle\left(\mathbb{R}^{m}\right)$ be a behavior strategy that maps a possible valuation to a distribution over bid vectors. For a valuation $v_{i}$, let $\Gamma\left(s_{i}, v_{i}\right)$ be the support of $s_{i}\left(v_{i}\right)$; that is, $\Gamma\left(s_{i}, v_{i}\right)$ gives the set of bid vectors that $i$ plays with positive probability. Let $f_{-i}\left(\boldsymbol{v}_{-i}\right)=\prod_{k \neq i} f_{k}\left(v_{k}\right)$ give the probability that the drawn other-agent valuations are $\boldsymbol{v}_{-i}$. The induced probability distribution over other-agent bids is given by

$$
\Psi\left(\boldsymbol{b}_{-i}\right)=\int_{\boldsymbol{v}_{-i} \in\left\{\boldsymbol{v}_{-i}^{\prime} \in \boldsymbol{V}_{-i} \mid \forall k \neq i, \boldsymbol{b}_{k} \in \Gamma\left(s_{i}, v_{k}^{\prime}\right)\right\}} f_{-i}\left(\boldsymbol{v}_{-i}\right) d \boldsymbol{v}_{-i} .
$$

For the purpose of the proofs, we introduce notation for additional marginal distributions. Let $\Psi_{\boldsymbol{Q}, \boldsymbol{H}}(\boldsymbol{q}, \boldsymbol{h})$ be the probability other agents place highest bids $q$ and that the number of highest bidders for each good is $h$,

$$
\Psi_{\boldsymbol{Q}, \boldsymbol{H}}(\boldsymbol{q}, \boldsymbol{h})=\int_{\boldsymbol{b}_{-i} \in\left\{\boldsymbol{b}_{-i} \mid \forall j \in \mathcal{X}, \max _{k \neq i}\left\{b_{k}^{j}\right\}=q^{j} \wedge \#\left\{k \neq i \mid b_{k}^{j}=q^{j}\right\}=h^{j}\right\}} \Psi\left(\boldsymbol{b}_{-i}\right) d \boldsymbol{b}_{-i} .
$$

Let $\Psi_{Q}(\boldsymbol{q})$ be the probability that other agents place highest bids $\boldsymbol{q}$ (marginalizing over $\boldsymbol{h}$ ): ${ }^{9}$

$$
\Psi_{Q}(\boldsymbol{q})=\sum_{\boldsymbol{h} \in \mathbb{N}_{+}^{m}} \Psi_{\boldsymbol{Q}, \boldsymbol{H}}(\boldsymbol{q}, \boldsymbol{h})
$$

\footnotetext{
${ }^{8}$ We introduce this notation because all of the preceding allocation rules can be expressed in this form, simplifying some of the proofs.

${ }^{9}$ Note that $\Pi$ from $\S 4$ is the cumulative form of this function.
} 
Similarly, let $\Psi_{\boldsymbol{H}}(\boldsymbol{h})$ be the probability that the number of highest bidders for each good is $\boldsymbol{h}$ (marginalizing over $\boldsymbol{q})$ :

$$
\Psi_{\boldsymbol{H}}(\boldsymbol{h})=\int_{\boldsymbol{q} \in \mathbb{R}_{+}^{m}} \Psi_{\boldsymbol{Q}, \boldsymbol{H}}(\boldsymbol{q}, \boldsymbol{h}) d \boldsymbol{q}
$$

Utility Agent $i$ 's expected utility (over possible allocations) for bidding $\boldsymbol{b}_{i}$ when other agents bid $\boldsymbol{b}_{-i}$ under allocation rule $w$ is

$$
u_{i}^{w}\left(\boldsymbol{b}_{i}, \boldsymbol{b}_{-i}\right)=\sum_{X \subseteq \mathcal{X}} w\left(X, \boldsymbol{b}_{i}, \boldsymbol{b}_{-i}\right)\left(v_{i}(X)-\psi^{X}\left(\boldsymbol{b}_{i}, \boldsymbol{b}_{-i}\right)\right) .
$$

Given a distribution over other-agent bids $\Psi$, agent $i$ 's expected utility (over other-agent bids and possible allocations) under allocation rule $w$ is

$$
U_{i}^{w}\left(\boldsymbol{b}_{i}, \Psi\right)=\mathbb{E}_{\boldsymbol{b}_{-i} \sim \Psi}\left[u_{i}^{w}\left(\boldsymbol{b}_{i}, \boldsymbol{b}_{-i}\right)\right] .
$$

For readability, in the remainder of this section, we suppress $\Psi$ and drop the agent subscripts from $U_{i}^{w}\left(\boldsymbol{b}_{i}, \Psi\right)$, so that agent $i$ 's expected utility is given by $U^{w}(\boldsymbol{b})$.

\section{A.2 Overview of Theorems}

Figure 5 outlines the main theorems and proofs. An arrow from statement $A$ to statement $B$ indicates that $B$ 's proof depends on $A$. Note the vertical symmetry in the figure. The proofs for statements on the right side of the figure (outlined in red) are nearly identical to the proofs for the corresponding statements on the left side. Also note that some of the statements in Figure 5 use the symbol $\approx$ to improve readability. For all such statements, the expression $U_{1} \approx U_{2}$ more specifically means that $\left|U_{1}-U_{2}\right| \leq \tau$, for all $\tau>0$.

Lemma 6 states that, given agent $i$ 's bids $\boldsymbol{b}$, highest other-agent bids $\boldsymbol{q}$, and a vector $\boldsymbol{p}=\left(p^{1} \ldots, p^{m}\right)$ specifying the probability $i$ wins good $j$ under a tie for that good, the probability agent $i$ wins bundle $X$ under allocation rule $w$ is a convex combination of the probabilities $i$ wins bundle $X$ under each approximate allocation rule with deterministic tiebreakers. This property holds for any allocation rule $w$ in which the winner is determined independently for each good. Note that, for true allocation rule $w^{*}(X, \boldsymbol{b}, \boldsymbol{q}, \boldsymbol{h})$, the lemma holds by defining $\boldsymbol{p}$ such that the probability of winning good $j$ is $p^{j}=1 /\left(h^{j}+1\right)$. Similarly, the lemma holds for an approximate allocation rule $\hat{w}_{p}(X, \boldsymbol{b}, \boldsymbol{q})$ by defining $\boldsymbol{p}$ to be $(p, \ldots, p)$ (i.e., the same fixed probability of winning a tie for each good).

Assumption 7 is our key condition, stating that the highest other-agent bids $q$ and the number of agents $h$ placing highest bids are independent.

Lemmas 8 and 9 use Lemma 6 to show that expected utility $U^{w^{*}}(\boldsymbol{b})$ under the true allocation rule $w^{*}$ and expected utility $U^{\hat{w}_{p}}(\boldsymbol{b})$ under an approximate allocation rule $\hat{w}_{p}$ are both convex combinations of expected utility under approximate allocation rules with deterministic tiebreakers $\widetilde{w}_{X}$. For the true allocation rule $w^{*}$, the result depends on Assumption 7.

Propositions 10 and 11 state that agent $i$ 's expected utility under the true allocation rule $w^{*}$ or approximate allocation rule $\hat{w}_{p}$, when $i$ bids optimally with respect to that allocation rule, is nearly the same as $i$ 's expected utility for placing those bids under any approximate allocation rule with deterministic tiebreakers.

Corollaries 12 and 13 state that, when agent $i$ places bids $b^{*}$ or $\hat{b}^{*}$ (i.e., when $i$ bids optimally with respect to $w^{*}$ or $\hat{w}_{p}$ ), $i$ 's expected utility is nearly the same for those bids under any approximate allocation rule with deterministic tiebreakers $\widetilde{w}$.

Proposition 14 states that, when placing bids $b^{*}$ that are optimal with respect to the true allocation rule $w^{*}$, the expected utility $U^{\hat{w}_{p}}\left(\boldsymbol{b}^{*}\right)$ under the approximate allocation rule is nearly the same as the expected 


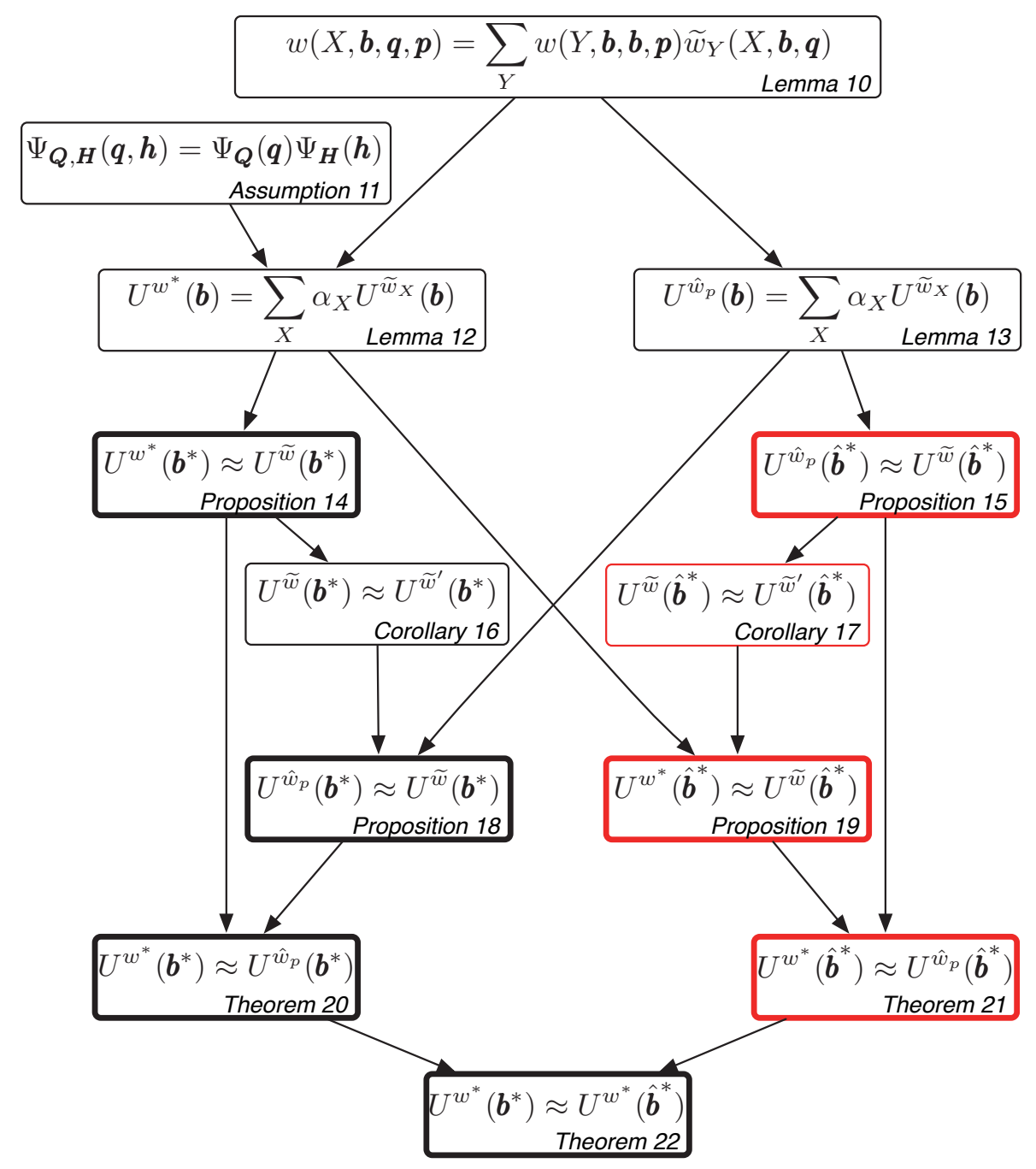

Figure 5: Proof overview.

utility $U^{\widetilde{w}}\left(\boldsymbol{b}^{*}\right)$ under any approximate allocation rule with deterministic tiebreakers. Similarly, Proposition 15 states that, when placing bids $\hat{b}^{*}$ that are optimal with respect to the approximate allocation rule $\hat{w}_{p}$, the expected utility $U^{w *}\left(\hat{\boldsymbol{b}}^{*}\right)$ under the true allocation rule is nearly the same as the expected utility $U^{\widetilde{w}}\left(\hat{\boldsymbol{b}}^{*}\right)$ under any approximate allocation rule with deterministic tiebreakers.

Theorem 16 states that agent $i$ 's expected utility $U^{w^{*}}\left(\boldsymbol{b}^{*}\right)$ under the true allocation rule $w^{*}$ when $i$ bids optimally with respect to the true allocation rule is nearly equal to $i$ 's expected utility $U^{\hat{w}_{p}}\left(\boldsymbol{b}^{*}\right)$ under the approximate allocation rule. Theorem 17 makes the same statement for bids $\hat{\boldsymbol{b}}_{i}^{*}$ that are optimal with respect to the approximate allocation rule.

Finally, Theorem 18 states that agent $i$ 's expected utility $U^{w^{*}}(\boldsymbol{b})$ under the true allocation rule $w^{*}$ is nearly the same whether it bidding optimally with respect to the true allocation rule $w^{*}$ or the approximate allocation rule $\hat{w}_{p}$. This theorem represents our bottom line: absent a dependence of highest other-agent bid on degree of ties, ignoring ties entails only infinitesimal regret. 


\section{A.3 Proofs}

Lemma 6. $w(X, \boldsymbol{b}, \boldsymbol{q}, \boldsymbol{p})=\sum_{Y} w(Y, \boldsymbol{b}, \boldsymbol{b}, \boldsymbol{p}) \widetilde{w}_{Y}(X, \boldsymbol{b}, \boldsymbol{q})$

Proof. Let $p^{j}$ be an element of $\boldsymbol{p}$ giving the probability agent $i$ wins good $j$ given that $i$ ties for that good's highest bid. Its complement, $\bar{p}^{j}=1-p^{j}$, is the probability of losing good $j$ given a tie.

We start by introducing notation that allows us to express the equation for winning bundle $X$ under allocation rule $w$ without conditionals in the definition. Given $i$ 's bids $\boldsymbol{b}$, highest other-agent bids $\boldsymbol{q}$, and a bundle of goods $X \subseteq \mathcal{X}$, let $\left(T^{X}, T^{\bar{X}}, W^{X}, W^{\bar{X}}, L^{X}, L^{\bar{X}}\right)$ be the following partition over all goods in $\mathcal{X}$ :

- $T^{X}(\boldsymbol{b}, \boldsymbol{q}, X)=\left\{j: b^{j}=q^{j} \wedge j \in X\right\}$ : the set of goods in $X$ for which agent $i$ tied for highest bid.

- $T^{\bar{X}}(\boldsymbol{b}, \boldsymbol{q}, X)=\left\{j: b^{j}=q^{j} \wedge j \notin X\right\}$ : the set of goods not in $X$ for which agent $i$ tied for highest bid.

- $W^{X}(\boldsymbol{b}, \boldsymbol{q}, X)=\left\{j: b^{j}>q^{j} \wedge j \in X\right\}$ : the set of goods in $X$ for which agent $i$ bid higher than the highest other-agent bid.

- $W^{\bar{X}}(\boldsymbol{b}, \boldsymbol{q}, X)=\left\{j: b^{j}>q^{j} \wedge j \notin X\right\}$ : the set of goods not in $X$ for which agent $i$ bid higher than the highest other-agent bid.

- $L^{X}(\boldsymbol{b}, \boldsymbol{q}, X)=\left\{j: b^{j}<q^{j} \wedge j \in X\right\}$ : the set of goods in $X$ for which agent $i$ bid lower than the highest other-agent bid.

- $L^{\bar{X}}(\boldsymbol{b}, \boldsymbol{q}, X)=\left\{j: b^{j}<q^{j} \wedge j \notin X\right\}$ : the set of goods not in $X$ for which agent $i$ bid lower than the highest other-agent bid.

Let $\bar{T}(\boldsymbol{b}, \boldsymbol{q}, X)=\mathcal{X} \backslash\left(T^{X}(\boldsymbol{b}, \boldsymbol{q}, X) \cup T^{\bar{X}}(\boldsymbol{b}, \boldsymbol{q}, X)\right)$ be the set of goods for which agent $i$ did not tie.

Finally, given a set of goods $Y$ for which all tiebreakers are won by agent $i$, we further partition $T^{X}$ into $\left(T^{X, Y}, T^{X, \bar{Y}}\right)$ and $T^{\bar{X}}$ into $\left(T^{\bar{X}, Y}, T^{\bar{X}, \bar{Y}}\right)$ as follows:

- $T^{X, Y}(\boldsymbol{b}, \boldsymbol{q}, X, Y)=\left\{j: b^{j}=q^{j} \wedge j \in X \wedge j \in Y\right\}$

- $T^{X, \bar{Y}}(\boldsymbol{b}, \boldsymbol{q}, X, Y)=\left\{j: b^{j}=q^{j} \wedge j \in X \wedge j \notin Y\right\}$

- $T^{\bar{X}, Y}(\boldsymbol{b}, \boldsymbol{q}, X, Y)=\left\{j: b^{j}=q^{j} \wedge j \notin X \wedge j \in Y\right\}$

- $T^{\bar{X}, \bar{Y}}(\boldsymbol{b}, \boldsymbol{q}, X, Y)=\left\{j: b^{j}=q^{j} \wedge j \notin X \wedge j \notin Y\right\}$

To simplify notation, we treat the arguments associated with the above sets as implicit. 


$$
\begin{aligned}
& w(X, \boldsymbol{b}, \boldsymbol{q}, \boldsymbol{p})=\prod_{j \in T^{X}} p^{j} \prod_{j \in T^{\bar{X}}} \bar{p}^{j} \prod_{j \in W^{X}} 1 \prod_{j \in W^{\bar{X}}} 0 \prod_{j \in L^{X}} 0 \prod_{j \in L^{\bar{X}}} 1 \\
& =\prod_{j \in T^{X}} p^{j} \prod_{j \in T^{\bar{X}}} \bar{p}^{j} \prod_{j \in W^{X}} 1 \prod_{j \in W^{\bar{X}}} 0 \prod_{j \in L^{X}} 0 \prod_{j \in L^{\bar{X}}} 1 \sum_{Y \subseteq \bar{T}} \prod_{j \in Y} p^{j} \prod_{j \in \bar{T} \backslash Y} \bar{p}^{j} \\
& =\prod_{j \in W^{X}} 1 \prod_{j \in W^{\bar{X}}} 0 \prod_{j \in L^{X}} 0 \prod_{j \in L^{\bar{X}}} 1 \sum_{Y \subseteq \bar{T}} \prod_{j \in T^{X}} p^{j} \prod_{j \in Y} p^{j} \prod_{j \in \bar{T} \backslash Y} \bar{p}^{j} \prod_{j \in T^{\bar{X}}} \bar{p}^{j} \\
& =\prod_{j \in W^{X}} 1 \prod_{j \in W^{\bar{X}}} 0 \prod_{j \in L^{X}} 0 \prod_{j \in L^{\bar{X}}} 1 \sum_{\substack{Y \subseteq \mathcal{X} \text { s.t. } \\
j \in T^{X} \leftrightarrow j \in Y \wedge \\
j \notin T^{X} \leftrightarrow j \notin Y}} \prod_{j \in Y} p^{j} \prod_{j \in \notin Y} \bar{p}^{j} \\
& =\prod_{j \in W^{X}} 1 \prod_{j \in W^{\bar{X}}} 0 \prod_{j \in L^{X}} 0 \prod_{j \in L^{\bar{X}}} 1 \sum_{Y \subseteq \mathcal{X}} \prod_{j \in T^{X}, Y} 1 \prod_{j \in T^{X, \bar{Y}}} 0 \prod_{j \in T^{\bar{X}}, Y} 0 \prod_{j \in T^{\bar{X}, \bar{Y}}} 1 \prod_{j \in Y} p^{j} \prod_{j \in \notin Y} \bar{p}^{j}
\end{aligned}
$$

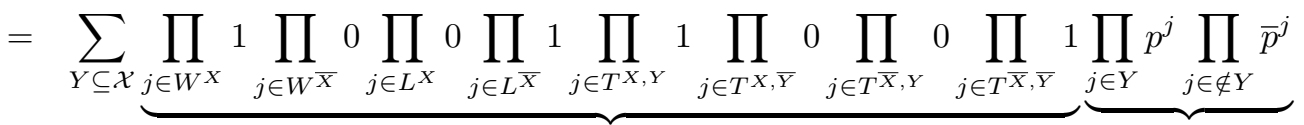

$$
\begin{aligned}
& =\sum_{Y \subseteq \mathcal{X}} \widetilde{w}_{Y}(X, \boldsymbol{b}, \boldsymbol{q}) w(Y, \boldsymbol{b}, \boldsymbol{b}, \boldsymbol{p})
\end{aligned}
$$

Equation (8) is the definition of $w$, replacing the conditionals in the allocation rule with indicator variables. (9) holds because $\sum_{Y \subseteq \bar{T}} \prod_{j \in Y} p^{j} \prod_{j \in \bar{T} \backslash Y} \bar{p}^{j}=1$. Rearranging terms yields (10), and (11) by combining some of the products on the right side of the summation (note that the summation is over the same number of elements as in the previous equation). We get (12) by replacing conditions from the summation with additional indicator variables, (13) by rearranging terms, and finally (14) by substituting in the definitions of $\widetilde{w}_{Y}$ and $w$.

Example 1. $X=\{1\}, \boldsymbol{b}=(10,10), \boldsymbol{q}=(10,11)$, and $\boldsymbol{p}=\left(\frac{1}{10}, \frac{1}{4}\right)$.

$$
\begin{aligned}
w(X, \boldsymbol{b}, \boldsymbol{q}, \boldsymbol{p})= & \sum_{Y} w(Y, \boldsymbol{b}, \boldsymbol{b}, \boldsymbol{p}) \widetilde{w}_{Y}(X, \boldsymbol{b}, \boldsymbol{q}) \\
= & w(\{\}, \boldsymbol{b}, \boldsymbol{b}, \boldsymbol{p}) \widetilde{w}_{\{\}}(X, \boldsymbol{b}, \boldsymbol{q})+w(\{1\}, \boldsymbol{b}, \boldsymbol{b}, \boldsymbol{p}) \widetilde{w}_{\{1\}}(X, \boldsymbol{b}, \boldsymbol{q})+ \\
& w(\{2\}, \boldsymbol{b}, \boldsymbol{b}, \boldsymbol{p}) \widetilde{w}_{\{2\}}(X, \boldsymbol{b}, \boldsymbol{q})+w(\{1,2\}, \boldsymbol{b}, \boldsymbol{b}, \boldsymbol{p}) \widetilde{w}_{\{1,2\}}(X, \boldsymbol{b}, \boldsymbol{q}) \\
= & \left(\frac{9}{10} \cdot \frac{3}{4}\right) 0+\left(\frac{1}{10} \cdot \frac{3}{4}\right) 1+ \\
& \left(\frac{9}{10} \cdot \frac{1}{4}\right) 0+\left(\frac{1}{10} \cdot \frac{1}{4}\right) 1 \\
= & \frac{1}{10}\left(\frac{3}{4}+\frac{1}{4}\right) \\
= & \frac{1}{10}
\end{aligned}
$$

Note that $\widetilde{w}_{Y}(X, \boldsymbol{b}, \boldsymbol{q})$ acts as an indicator, evaluating to 1 if ties are broken so that bundle $X$ is exactly won and evaluating to 0 otherwise. In the example, bundle $X$ is won only under approximate allocation 
rules with deterministic tiebreakers that win good 1 in the event of a tie (i.e., $Y=\{1\}$ and $Y=\{1,2\}$ ). Whether or not good 2 is won in the event of a tie is irrelevant.

Also note that $w(Y, \boldsymbol{b}, \boldsymbol{b}, \boldsymbol{p})$ is the probability of winning bundle $Y$ if the agent ties for every good. The irrelevant goods are effectively marginalized.

Assumption 7. The distribution over highest other-agent bids and the distribution over the number of agents placing the same highest bids are independent: $\Psi_{\boldsymbol{Q}, \boldsymbol{H}}(\boldsymbol{q}, \boldsymbol{h})=\Psi_{\boldsymbol{Q}}(\boldsymbol{q}) \Psi_{\boldsymbol{H}}(\boldsymbol{h})$.

Lemma 8. Given Assumption 7, $U^{w^{*}}(\boldsymbol{b})=\sum_{Y \subseteq \mathcal{X}} \alpha_{Y} U^{\widetilde{w}_{Y}}(\boldsymbol{b}) \quad\left(\alpha_{Y} \geq 0, \sum_{Y \subseteq \mathcal{X}} \alpha_{Y}=1\right)$.

Proof. First, we derive an expression for expected utility $U^{\widetilde{w}_{Y}}(\boldsymbol{b})$ under approximate allocation rule $\widetilde{w}_{Y}$ with deterministic tiebreakers.

$$
\begin{aligned}
U^{\widetilde{w}_{Y}(\boldsymbol{b})} & =\int_{\boldsymbol{q}} \sum_{\boldsymbol{h}} \Psi_{\boldsymbol{Q}, \boldsymbol{H}}(\boldsymbol{q}, \boldsymbol{h}) \sum_{X \subseteq \mathcal{X}} \widetilde{w}_{Y}(X, \boldsymbol{b}, \boldsymbol{q})\left[v(X)-\psi^{X}(\boldsymbol{b}, \boldsymbol{q})\right] d \boldsymbol{q} \\
& =\int_{\boldsymbol{q}} \sum_{\boldsymbol{h}} \Psi_{\boldsymbol{Q}}(\boldsymbol{q}) \Psi_{\boldsymbol{H}}(\boldsymbol{h}) \sum_{X \subseteq \mathcal{X}} \widetilde{w}_{Y}(X, \boldsymbol{b}, \boldsymbol{q})\left[v(X)-\psi^{X}(\boldsymbol{b}, \boldsymbol{q})\right] d \boldsymbol{q} \\
& =\int_{\boldsymbol{q}} \Psi_{\boldsymbol{Q}}(\boldsymbol{q}) \sum_{X \subseteq \mathcal{X}} \widetilde{w}_{Y}(X, \boldsymbol{b}, \boldsymbol{q})\left[v(X)-\psi^{X}(\boldsymbol{b}, \boldsymbol{q})\right] \sum_{\boldsymbol{h}} \Psi_{\boldsymbol{H}}(\boldsymbol{h}) d \boldsymbol{q} \\
& =\int_{\boldsymbol{q}} \Psi_{\boldsymbol{Q}}(\boldsymbol{q}) \sum_{X \subseteq \mathcal{X}} \widetilde{w}_{Y}(X, \boldsymbol{b}, \boldsymbol{q})\left[v(X)-\psi^{X}(\boldsymbol{b}, \boldsymbol{q})\right] d \boldsymbol{q}
\end{aligned}
$$

Equation (15) is the definition of expected utility. Applying Assumption 7 yields (16). Rearranging terms (17) and applying $\sum_{\boldsymbol{h}} \Psi_{\boldsymbol{H}}(\boldsymbol{h})=1$ produces the final form (18).

A similar derivation produces an expression for expected utility $U^{w^{*}}(\boldsymbol{b})$ under the true allocation rule $w^{*}$.

$$
\begin{aligned}
U^{w^{*}}(\boldsymbol{b}) & =\int_{\boldsymbol{q}} \sum_{\boldsymbol{h}} \Psi_{\boldsymbol{Q}, \boldsymbol{H}}(\boldsymbol{q}, \boldsymbol{h}) \sum_{X \subseteq \mathcal{X}} w^{*}(X, \boldsymbol{b}, \boldsymbol{q})\left[v(X)-\psi^{X}(\boldsymbol{b}, \boldsymbol{q})\right] d \boldsymbol{q} \\
& =\int_{\boldsymbol{q}} \sum_{\boldsymbol{h}} \Psi_{\boldsymbol{Q}, \boldsymbol{H}}(\boldsymbol{q}, \boldsymbol{h}) \sum_{X \subseteq \mathcal{X}} \sum_{Y \subseteq \mathcal{X}} w^{*}(Y, \boldsymbol{b}, \boldsymbol{b}, \boldsymbol{h}) \widetilde{w}_{Y}(X, \boldsymbol{b}, \boldsymbol{q})\left[v(X)-\psi^{X}(\boldsymbol{b}, \boldsymbol{q})\right] d \boldsymbol{q} \\
& =\int_{\boldsymbol{q}} \sum_{\boldsymbol{h}} \Psi_{\boldsymbol{Q}}(\boldsymbol{q}) \Psi_{\boldsymbol{H}}(\boldsymbol{h}) \sum_{X \subseteq \mathcal{X}} \sum_{Y \subseteq \mathcal{X}} w^{*}(Y, \boldsymbol{b}, \boldsymbol{b}, \boldsymbol{h}) \widetilde{w}_{Y}(X, \boldsymbol{b}, \boldsymbol{q})\left[v(X)-\psi^{X}(\boldsymbol{b}, \boldsymbol{q})\right] d \boldsymbol{q} \\
& =\sum_{Y \subseteq \mathcal{X}} \sum_{\boldsymbol{h}} \Psi_{\boldsymbol{H}}(\boldsymbol{h}) w^{*}(Y, \boldsymbol{b}, \boldsymbol{b}, \boldsymbol{h}) \int_{\boldsymbol{q}} \Psi_{\boldsymbol{Q}}(\boldsymbol{q}) \sum_{X \subseteq \mathcal{X}} \widetilde{w}_{Y}(X, \boldsymbol{b}, \boldsymbol{q})\left[v(X)-\psi^{X}(\boldsymbol{b}, \boldsymbol{q})\right] d \boldsymbol{q} \\
& =\sum_{Y \subseteq \mathcal{X}} \sum_{\boldsymbol{h}} \Psi_{\boldsymbol{H}}(\boldsymbol{h}) w^{*}(Y, \boldsymbol{b}, \boldsymbol{b}, \boldsymbol{h}) U^{\widetilde{w}_{Y}}(\boldsymbol{b}) \\
& =\sum_{Y \subseteq \mathcal{X}} \alpha_{Y} U^{\widetilde{w}_{Y}}(\boldsymbol{b})
\end{aligned}
$$

Starting with the definition of expected utility (19), we apply Lemma 6 to get (20), and Assumption 7 to get (21). Rearranging terms (22), and substituting in the simplified form (18) for $U^{\widetilde{w}_{Y}}(\boldsymbol{b})$ yields (23). The last step (24) follows from $\alpha_{Y}=\sum_{\boldsymbol{h}} \Psi_{\boldsymbol{H}}(\boldsymbol{h}) w^{*}(Y, \boldsymbol{b}, \boldsymbol{b}, \boldsymbol{h})$. All that remains is to show that these $\alpha_{Y}$ values satisfy the properties stated in Lemma 8 . For all $Y \subseteq \mathcal{X}$, we have $\alpha_{Y} \geq 0$ since both components 
$\Psi_{\boldsymbol{H}}(\boldsymbol{h})$ and $w^{*}(Y, \boldsymbol{b}, \boldsymbol{b}, \boldsymbol{h})$ are probabilities and thus non-negative for all $\boldsymbol{h}, Y$, and $\boldsymbol{b}$. Further, $\sum_{Y \subseteq \mathcal{X}} \alpha_{Y}=$ $\sum_{\boldsymbol{h}} \Psi_{\boldsymbol{H}}(\boldsymbol{h}) \sum_{Y \subseteq \mathcal{X}} w^{*}(Y, \boldsymbol{b}, \boldsymbol{b}, \boldsymbol{h})=1$, since the distribution over bundles $\sum_{Y \subseteq \mathcal{X}} w^{*}(Y, \boldsymbol{b}, \boldsymbol{b}, \boldsymbol{h})$ sums to 1 for all values of $\bar{h}$ and $\boldsymbol{b}$, and $\sum_{\boldsymbol{h}} \Psi_{\boldsymbol{H}}(\boldsymbol{h})$ similarly sums to $1 .{ }^{10}$

The following states that agent $i$ 's expected utility under the approximate allocation rule is a convex combination its expected utilities under the approximate allocation rules with deterministic tiebreakers.

Lemma 9. $U^{\hat{w}_{p}}(\boldsymbol{b})=\sum_{X \subseteq \mathcal{X}} \alpha_{X} U^{\widetilde{w}_{X}}(\boldsymbol{b}) \quad\left(\forall p \in[0,1], \alpha_{X} \geq 0, \sum_{X \subseteq \mathcal{X}} \alpha_{X}=1\right)$.

Proof.

$$
\begin{aligned}
U^{\hat{w}_{p}}(\boldsymbol{b}) & =\int_{\boldsymbol{q}} \sum_{\boldsymbol{h}} \Psi_{\boldsymbol{Q}, \boldsymbol{H}}(\boldsymbol{q}, \boldsymbol{h}) \sum_{X \subseteq \mathcal{X}} \hat{w}_{p}(X, \boldsymbol{b}, \boldsymbol{q})\left[v(X)-\psi^{X}(\boldsymbol{b}, \boldsymbol{q})\right] d \boldsymbol{q} \\
& =\int_{\boldsymbol{q}} \sum_{\boldsymbol{h}} \Psi_{\boldsymbol{Q}, \boldsymbol{H}}(\boldsymbol{q}, \boldsymbol{h}) \sum_{X \subseteq \mathcal{X}} \sum_{Y \subseteq \mathcal{X}} \hat{w}_{p}(Y, \boldsymbol{b}, \boldsymbol{b}) \widetilde{w}_{Y}(X, \boldsymbol{b}, \boldsymbol{q})\left[v(X)-\psi^{X}(\boldsymbol{b}, \boldsymbol{q})\right] d \boldsymbol{q} \\
& =\sum_{Y \subseteq \mathcal{X}} \hat{w}_{p}(Y, \boldsymbol{b}, \boldsymbol{b}) \int_{\boldsymbol{q}} \sum_{\boldsymbol{h}} \Psi_{\boldsymbol{Q}, \boldsymbol{H}}(\boldsymbol{q}, \boldsymbol{h}) \sum_{X \subseteq \mathcal{X}} \widetilde{w}_{Y}(X, \boldsymbol{b}, \boldsymbol{q})\left[v(X)-\psi^{X}(\boldsymbol{b}, \boldsymbol{q})\right] d \boldsymbol{q} \\
& =\sum_{Y \subseteq \mathcal{X}} \hat{w}_{p}(Y, \boldsymbol{b}, \boldsymbol{b}) U^{\widetilde{w}_{Y}}(\boldsymbol{b}) \\
& =\sum_{Y \subseteq \mathcal{X}} \alpha_{Y} U^{\widetilde{w}_{Y}}(\boldsymbol{b})
\end{aligned}
$$

Equation (25) follows from application of Lemma 6 to the definition of $U^{\hat{w}_{p}}$. Rearranging terms and applying the definition of $U^{\widetilde{w}_{Y}}(\boldsymbol{b})$ (15) yields (26). The final form (27) is obtained with $\alpha_{Y}=\hat{w}_{p}(Y, \boldsymbol{b}, \boldsymbol{b})$. Note that $\hat{w}_{p}(Y, \boldsymbol{b}, \boldsymbol{b}) \geq 0$ when $p \in[0,1]$ since by definition (7) it is a product of values between 0 and 1 . Since $\sum_{Y} \hat{w}_{p}(Y, \boldsymbol{b}, \boldsymbol{b})=1$, the requisite $\alpha_{Y}$ properties for the lemma are satisfied.

The following states that agent $i$ 's expected utility under the true allocation rule is nearly the same as its expected utility under any approximate allocation rule with deterministic tiebreakers, given it bids optimally with respect to $U^{w^{*}}$.

Proposition 10. Given Assumption 7, $\forall \tau>0, \widetilde{w} \in \widetilde{W},\left|U^{w^{*}}\left(\boldsymbol{b}^{*}\right)-U^{\widetilde{w}}\left(\boldsymbol{b}^{*}\right)\right| \leq \tau$.

Proof. Let $\widetilde{w} \in \widetilde{W}$ be an approximate allocation rule with deterministic tiebreakers. Let $\boldsymbol{b}^{* \rightarrow \widetilde{w}}$ be the optimal bid vector with its bids perturbed by an infinitesimally small amount so that ties are broken in favor of $\widetilde{w}$. Let $B_{-i}$ be the set of possible other-agent bids, and let $B_{-i}^{\prime} \subseteq B_{-i}$ be possible other-agent bids such that the highest other-agent bid for at least one good $j$ falls between $b_{i}^{* j}$ and $b_{i}^{* \rightarrow \widetilde{w} j}$.

We show that the bound holds in both possible orderings of expected utility.

\footnotetext{
${ }^{10}$ Intuitively, $w^{*}(Y, \boldsymbol{b}, \boldsymbol{b}, \boldsymbol{h})$ gives the probability of an agent winning bundle $Y$ when it ties for every good $j$ with $h^{j}$ other agents. According to the allocation rule, this probability does not depend on the particular value of $\boldsymbol{b}$.
} 
Case 1: $U^{w^{*}}\left(\boldsymbol{b}^{*}\right)-U^{\widetilde{w}}\left(\boldsymbol{b}^{*}\right) \leq 0$.

$$
\begin{aligned}
\left|U^{w^{*}}\left(\boldsymbol{b}^{*}\right)-U^{\widetilde{w}}\left(\boldsymbol{b}^{*}\right)\right| & \leq\left|U^{w^{*}}\left(\boldsymbol{b}^{* \rightarrow \widetilde{w}}\right)-U^{\widetilde{w}}\left(\boldsymbol{b}^{*}\right)\right| \\
& =\left|\int_{B_{-i}} \Psi\left(\boldsymbol{b}_{-i}\right) u^{w^{*}}\left(\boldsymbol{b}^{* \rightarrow \widetilde{w}}, \boldsymbol{b}_{-i}\right) d \boldsymbol{b}_{-i}-\int_{B_{-i}} \Psi\left(\boldsymbol{b}_{-i}\right) u^{\widetilde{w}}\left(\boldsymbol{b}^{*}, \boldsymbol{b}_{-i}\right) d \boldsymbol{b}_{-i}\right| \\
& =\left|\int_{B_{-i}} \Psi\left(\boldsymbol{b}_{-i}\right)\left[u^{w^{*}}\left(\boldsymbol{b}^{* \rightarrow \widetilde{w}}, \boldsymbol{b}_{-i}\right)-u^{\widetilde{w}}\left(\boldsymbol{b}^{*}, \boldsymbol{b}_{-i}\right)\right] d \boldsymbol{b}_{-i}\right| \\
& =\mid \int_{B_{-i} \backslash B_{-i}^{\prime}} \Psi\left(\boldsymbol{b}_{-i}\right)\left[u^{w^{*}}\left(\boldsymbol{b}^{* \rightarrow \widetilde{w}}, \boldsymbol{b}_{-i}\right)-u^{\widetilde{w}}\left(\boldsymbol{b}^{*}, \boldsymbol{b}_{-i}\right)\right] d \boldsymbol{b}_{-i}+ \\
& \int_{B_{-i}^{\prime}} \Psi\left(\boldsymbol{b}_{-i}\right)\left[u^{w^{*}}\left(\boldsymbol{b}^{* \rightarrow \widetilde{w}}, \boldsymbol{b}_{-i}\right)-u^{\widetilde{w}}\left(\boldsymbol{b}^{*}, \boldsymbol{b}_{-i}\right)\right] d \boldsymbol{b}_{-i} \mid \\
& =\left|\int_{B_{-i}^{\prime}} \Psi\left(\boldsymbol{b}_{-i}\right)\left[u^{w^{*}}\left(\boldsymbol{b}^{* \rightarrow \widetilde{w}}, \boldsymbol{b}_{-i}\right)-u^{\widetilde{w}}\left(\boldsymbol{b}^{*}, \boldsymbol{b}_{-i}\right)\right] d \boldsymbol{b}_{-i}\right| \\
& \leq \tau
\end{aligned}
$$

Inequality (28) holds because $\boldsymbol{b}^{*}$ is optimal with respect to $U^{w^{*}}$. Successive steps substitute the definition of $U$ (29), combine like terms (30), and split the domain of the integral (32). The first term of (32) is zero, yielding (33). The final inequality (34) holds because the set $B_{-i}^{\prime}$ can be made arbitrarily small.

Case 2: $U^{w^{*}}\left(\boldsymbol{b}^{*}\right)-U^{\widetilde{w}}\left(\boldsymbol{b}^{*}\right)>0$.

We start by applying Lemma 8 , which enables us to express $U^{w^{*}}\left(\boldsymbol{b}^{*}\right)$ as a linear combination of the expected utilities of approximate allocation rules with deterministic tiebreakers.

$$
\begin{aligned}
& \left|U^{w^{*}}\left(\boldsymbol{b}^{*}\right)-U^{\widetilde{w}_{Y}}\left(\boldsymbol{b}^{*}\right)\right|=\left|U^{w^{*}}\left(\boldsymbol{b}^{*}\right)-\frac{U^{w^{*}}\left(\boldsymbol{b}^{*}\right)-\sum_{X \neq Y} \alpha_{X} U^{\widetilde{w}_{X}}\left(\boldsymbol{b}^{*}\right)}{\alpha_{Y}}\right| \\
& \leq\left|U^{w^{*}}\left(\boldsymbol{b}^{*}\right)-\frac{U^{w^{*}}\left(\boldsymbol{b}^{*}\right)-\sum_{X \neq Y} \alpha_{X}\left(U^{w^{*}}\left(\boldsymbol{b}^{*}\right)+\tau_{1}\right)}{\alpha_{Y}}\right| \\
& =\left|U^{w^{*}}\left(\boldsymbol{b}^{*}\right)-\frac{U^{w^{*}}\left(\boldsymbol{b}^{*}\right)-\left(U^{w^{*}}\left(\boldsymbol{b}^{*}\right)+\tau_{1}\right)\left(1-\alpha_{Y}\right)}{\alpha_{Y}}\right| \\
& =\left|U^{w^{*}}\left(\boldsymbol{b}^{*}\right)-\frac{U^{w^{*}}\left(\boldsymbol{b}^{*}\right)-\left[U^{w^{*}}\left(\boldsymbol{b}^{*}\right)-\alpha_{Y} U^{w^{*}}\left(\boldsymbol{b}^{*}\right)+\tau_{1}-\alpha_{Y} \tau_{1}\right]}{\alpha_{Y}}\right| \\
& =\left|U^{w^{*}}\left(\boldsymbol{b}^{*}\right)-\frac{\alpha_{Y} U^{w^{*}}\left(\boldsymbol{b}^{*}\right)-\tau_{1}+\alpha_{Y} \tau_{1}}{\alpha_{Y}}\right| \\
& =\left|U^{w^{*}}\left(\boldsymbol{b}^{*}\right)-\frac{\alpha_{Y}\left(U^{w^{*}}\left(\boldsymbol{b}^{*}\right)-\frac{\tau_{1}}{\alpha_{Y}}+\tau_{1}\right)}{\alpha_{Y}}\right| \\
& =\left|U^{w^{*}}\left(\boldsymbol{b}^{*}\right)-\left(U^{w^{*}}\left(\boldsymbol{b}^{*}\right)-\frac{\tau_{1}}{\alpha_{Y}}+\tau_{1}\right)\right| \\
& =\left|\tau_{1}-\frac{\tau_{1}}{\alpha_{Y}}\right|
\end{aligned}
$$


Inequality (35) follows from Case 1 of this proof. By definition of a convex combination, $\sum_{X \neq Y} \alpha_{X}=$ $1-\alpha_{Y}$, which gives us (36). All subsequent steps are the result of simplifying terms.

Proposition 11. $\forall \tau>0, \widetilde{w} \in \widetilde{W},\left|U^{\hat{w}_{p}}\left(\hat{\boldsymbol{b}}^{*}\right)-U^{\widetilde{w}}\left(\hat{\boldsymbol{b}}^{*}\right)\right| \leq \tau$.

Proof. The reasoning is exactly the same as that of Proposition 10 except that, throughout the proof, $\boldsymbol{b}_{i}^{*}$ is replaced by $\hat{\boldsymbol{b}}^{*}$ and $w^{*}$ by $\hat{w}_{p}$.

The following states that agent $i$ 's expected utility is nearly the same under any approximate allocation rule with deterministic tiebreakers, given it bids optimally.

Corollary 12. Given Assumption $7, \forall \tau>0, \widetilde{w}, \widetilde{w}^{\prime} \in \widetilde{W},\left|U^{\widetilde{w}}\left(\boldsymbol{b}^{*}\right)-U^{\widetilde{w}^{\prime}}\left(\boldsymbol{b}^{*}\right)\right| \leq \tau$.

Proof. Case 1: $U^{\widetilde{w}}\left(\boldsymbol{b}^{*}\right)-U^{\widetilde{w}^{\prime}}\left(\boldsymbol{b}^{*}\right) \geq 0$

$$
\begin{aligned}
\left|U^{\widetilde{w}}\left(\boldsymbol{b}^{*}\right)-U^{\widetilde{w}^{\prime}}\left(\boldsymbol{b}^{*}\right)\right| & \leq\left|\left[U^{w^{*}}\left(\boldsymbol{b}^{*}\right)+\tau / 2\right]-\left[U^{w^{*}}\left(\boldsymbol{b}^{*}\right)-\tau / 2\right]\right| \\
& =\tau
\end{aligned}
$$

Case 2: $U^{\widetilde{w}}\left(\boldsymbol{b}^{*}\right)-U^{\widetilde{w}^{\prime}}\left(\boldsymbol{b}^{*}\right)<0$

$$
\begin{aligned}
\left|U^{\widetilde{w}}\left(\boldsymbol{b}^{*}\right)-U^{\widetilde{w}^{\prime}}\left(\boldsymbol{b}^{*}\right)\right| & \leq\left|\left[U^{w^{*}}\left(\boldsymbol{b}^{*}\right)-\tau / 2\right]-\left[U^{w^{*}}\left(\boldsymbol{b}^{*}\right)+\tau / 2\right]\right| \\
& =\tau
\end{aligned}
$$

Both (37) and (38) follow from Proposition 10 (which requires Assumption 7).

Corollary 13. $\forall \tau>0, \widetilde{w}, \widetilde{w}^{\prime} \in \widetilde{W},\left|U^{\widetilde{w}}\left(\hat{\boldsymbol{b}}^{*}\right)-U^{\widetilde{w}^{\prime}}\left(\hat{\boldsymbol{b}}^{*}\right)\right| \leq \tau$.

Proof. The reasoning is exactly the same as that of Corollary 12 except that, throughout the proof, $\boldsymbol{b}_{i}^{*}$ is replaced by $\hat{\boldsymbol{b}}^{*}$ and $w^{*}$ by $\hat{w}_{p}$.

The following states that agent $i$ 's expected utility under the approximate allocation rule is nearly the same as its expected utility under any approximate allocation rule with deterministic tiebreakers, given it bids optimally.

Proposition 14. Given Assumption 7, $\forall \tau>0, \widetilde{w} \in \widetilde{W},\left|U^{\hat{w}_{p}}\left(\boldsymbol{b}^{*}\right)-U^{\widetilde{w}}\left(\boldsymbol{b}^{*}\right)\right| \leq \tau$.

Proof. Let $\widetilde{w} \in \widetilde{W}$ be any approximate allocation rule with deterministic tiebreakers.

Case 1: $U^{\hat{w}_{p}}\left(\boldsymbol{b}^{*}\right)-U^{\widetilde{w}}\left(\boldsymbol{b}^{*}\right) \geq 0$.

$$
\begin{aligned}
\left|U^{\hat{w}_{p}}\left(\boldsymbol{b}^{*}\right)-U^{\widetilde{w}}\left(\boldsymbol{b}^{*}\right)\right| & =\left|\sum_{X \subseteq \mathcal{X}} \alpha_{X} U^{\widetilde{w}}\left(\boldsymbol{b}^{*}\right)-U^{\widetilde{w}}\left(\boldsymbol{b}^{*}\right)\right| \\
& \leq\left|\sum_{X \subseteq \mathcal{X}} \alpha_{X}\left(U^{\widetilde{w}}\left(\boldsymbol{b}^{*}\right)+\tau\right)-U^{\widetilde{w}}\left(\boldsymbol{b}^{*}\right)\right| \\
& =\left|\left(U^{\widetilde{w}}\left(\boldsymbol{b}^{*}\right)+\tau\right) \sum_{X \subseteq \mathcal{X}} \alpha_{X}-U^{\widetilde{w}}\left(\boldsymbol{b}^{*}\right)\right| \\
& =\tau
\end{aligned}
$$


Equation (39) is true by Lemma 9. Applying Corollary 12, and thereby invoking Assumption 7, yields (40). Rearranging terms, and simplifying the expression using $\sum_{X \subseteq \mathcal{X}} \alpha_{X}=1$ (by Lemma 9) produces the result (41).

Case 2: $U^{\hat{w}_{p}}\left(\boldsymbol{b}^{*}\right)-U^{\widetilde{w}}\left(\boldsymbol{b}^{*}\right)<0$.

$$
\begin{aligned}
\left|U^{\hat{w}_{p}}\left(\boldsymbol{b}^{*}\right)-U^{\widetilde{w}}\left(\boldsymbol{b}^{*}\right)\right| & =\left|\sum_{X \subseteq \mathcal{X}} \alpha_{X} U^{\widetilde{w}}\left(\boldsymbol{b}^{*}\right)-U^{\widetilde{w}}\left(\boldsymbol{b}^{*}\right)\right| \\
& \leq\left|\sum_{X \subseteq \mathcal{X}} \alpha_{X}\left(U^{\widetilde{w}}\left(\boldsymbol{b}^{*}\right)-\tau\right)-U^{\widetilde{w}}\left(\boldsymbol{b}^{*}\right)\right| \\
& =\left|\left(U^{\widetilde{w}}\left(\boldsymbol{b}^{*}\right)-\tau\right) \sum_{X \subseteq \mathcal{X}} \alpha_{X}-U^{\widetilde{w}}\left(\boldsymbol{b}^{*}\right)\right| \\
& =\tau
\end{aligned}
$$

The reasoning in each step of Case 2 is the same as in Case 1.

Proposition 15. Given Assumption 7, $\forall \tau>0, \widetilde{w} \in \widetilde{W},\left|U^{w^{*}}\left(\hat{\boldsymbol{b}}^{*}\right)-U^{\widetilde{w}}\left(\hat{\boldsymbol{b}}^{*}\right)\right| \leq \tau$.

Proof. The reasoning is similar to that of Proposition 14. The only difference is that the analog of (39) follows from Lemma 8 (which uses Assumption 7), and the analog of (40) holds by Corollary 13.

The following states that agent $i$ 's expected utility under the true tiebreaker rule is nearly the same as its expected utility under the approximated tiebreaker rule, given it bids optimally.

Theorem 16. Given Assumption 7, $\forall \tau>0, p \in[0,1],\left|U^{w^{*}}\left(\boldsymbol{b}^{*}\right)-U^{\hat{w}_{p}}\left(\boldsymbol{b}^{*}\right)\right| \leq \tau$.

Proof. Case 1: $U^{w^{*}}\left(\boldsymbol{b}^{*}\right)-U^{\hat{w}_{p}}\left(\boldsymbol{b}^{*}\right) \geq 0$.

$$
\begin{aligned}
\left|U^{w^{*}}\left(\boldsymbol{b}^{*}\right)-U^{\hat{w}_{p}}\left(\boldsymbol{b}^{*}\right)\right| & \leq\left|\left[U^{\widetilde{w}}\left(\boldsymbol{b}^{*}\right)+\tau / 2\right]-\left[U^{\widetilde{w}}\left(\boldsymbol{b}^{*}\right)-\tau / 2\right]\right| \\
& =\tau
\end{aligned}
$$

Inequality (42) follows from Propositions 10 and 14 (which both use Assumption 7).

Case 2: $U^{w^{*}}\left(\boldsymbol{b}^{*}\right)-U^{\hat{w}_{p}}\left(\boldsymbol{b}^{*}\right)<0$.

$$
\begin{aligned}
\left|U^{w^{*}}\left(\boldsymbol{b}^{*}\right)-U^{\hat{w}_{p}}\left(\boldsymbol{b}^{*}\right)\right| & \leq\left|\left[U^{\widetilde{w}}\left(\boldsymbol{b}^{*}\right)-\tau / 2\right]-\left[U^{\widetilde{w}}\left(\boldsymbol{b}^{*}\right)+\tau / 2\right]\right| \\
& =\tau
\end{aligned}
$$

Theorem 17. $\forall \tau>0, p \in[0,1],\left|U^{w^{*}}\left(\hat{\boldsymbol{b}}^{*}\right)-U^{\hat{w}_{p}}\left(\hat{\boldsymbol{b}}^{*}\right)\right| \leq \tau$.

Proof. The reasoning is exactly the same as for Theorem 16.

Theorem 18. Given Assumption $7, U\left(\boldsymbol{b}^{*}\right) \leq U\left(\hat{\boldsymbol{b}}^{*}\right)+\tau$, for any $\tau>0$. 
Proof. Let $\tau$ be any value greater than 0 .

$$
\begin{aligned}
U^{w^{*}}\left(\boldsymbol{b}^{*}\right) & \leq U^{\hat{w}_{p}}\left(\boldsymbol{b}^{*}\right)+\tau / 2 \\
& \leq U^{\hat{w}_{p}}\left(\hat{\boldsymbol{b}}^{*}\right)+\tau / 2 \\
& \left.\leq U^{w^{*}}\left(\hat{\boldsymbol{b}}^{*}\right)+\tau / 2\right)+\tau / 2 \\
& =U^{w^{*}}\left(\hat{\boldsymbol{b}}^{*}\right)+\tau
\end{aligned}
$$

Inequality (43) follows from Theorem 16 (which uses Assumption 7). The next step (44) follows because $U^{\hat{w}_{p}}\left(\boldsymbol{b}^{*}\right) \leq U^{\hat{w}_{p}}\left(\hat{\boldsymbol{b}}^{*}\right)$ by definition of $\hat{\boldsymbol{b}}^{*}$. Applying Theorem 17 yields (45).

\section{A.4 With Probabilistic Dependence, Ties Can Matter}

Proposition 19. Theorem 16 does not necessarily hold if Assumption 7 is violated. That is, it is not the case that $\left|U^{w^{*}}\left(\boldsymbol{b}^{*}\right)-U^{\hat{w}_{p}}\left(\boldsymbol{b}^{*}\right)\right| \leq \tau \quad(\forall \tau>0, \forall p \in[0,1])$.

Proof. Suppose there are $n$ bidders and $m=2$ goods. $\Psi$ is as follows:

- With probability .5 , one opponent bids $\boldsymbol{b}_{j}=(10,0)$ and every other opponent $k \neq j$ bids $\boldsymbol{b}_{k}=(0,0)$. Call this realized other-agent bid vector $\boldsymbol{b}_{-i}^{(A)}$.

- With probability .5, all $n$ bidders bid $\boldsymbol{b}_{k}=(10, C)$, where $C$ is high enough that winning good 2 gives $i$ negative expected utility. Call this realized other-agent bid vector $\boldsymbol{b}_{-i}^{(B)}$.

Let bidder $i$ 's valuation be such that $v_{i}(\{1,2\})=15$ and for any other bundle $X, v_{i}(X)=0$.

To find the optimal bid vector for bidder $i$, we need consider only three candidates: $\boldsymbol{b}_{i}=(10, \epsilon)$, $\boldsymbol{b}_{i}^{\prime}=(10+\epsilon, \epsilon), \boldsymbol{b}_{i}^{\prime \prime}=(10-\epsilon, \epsilon)$. Since payments are second-price, any other bid vector will result in the same utility as one of these three bids (or negative utility in the case of bidding more than $C$ for good 2).

Consider the expected utility $U_{i}^{w^{*}}\left(\boldsymbol{b}_{i}, \Psi\right)$ for each of these candidate bid vectors. For reference, recall the expected utility equation:

$$
U_{i}^{w}\left(\boldsymbol{b}_{i}, \Psi\right)=\mathbb{E}_{\boldsymbol{b}_{-i} \sim \Psi}\left[u_{i}^{w}\left(\boldsymbol{b}_{i}, \boldsymbol{b}_{-i}\right)\right]=\mathbb{E}_{\boldsymbol{b}_{-i} \sim \Psi}\left[\sum_{X \subseteq \mathcal{X}} w_{i}^{X}\left(\boldsymbol{b}_{i}, \boldsymbol{b}_{-i}\right)\left[v_{i}(X)-\psi_{i}^{X}\left(\boldsymbol{b}_{i}, \boldsymbol{b}_{-i}\right)\right]\right]
$$

- When $\boldsymbol{b}_{i}=(10, \epsilon)$, with .5 probability, $\boldsymbol{b}_{-i}^{(A)}$ occurs, in which case bidder $i$ wins both goods with probability .5 (receiving value 15 and paying 10), and wins only good 2 with probability .5 (receiving value 0 and paying 0 ). With .5 probability, $\boldsymbol{b}_{-i}^{(B)}$ occurs, in which case bidder $i$ wins only good 1 with probability $\frac{1}{n}$ (receiving value 0 and paying 10 ) and wins no goods with probability $\frac{n-1}{n}$ (receiving value 0 and paying 0$): U^{w^{*}}((10, \epsilon))=.5[.5(15-10)+.5(0)]+.5\left[\frac{1}{n}(-10)+\frac{n-1}{n}(0)\right]=1.25+$ $\frac{1}{2 n}(-10)$.

- When $\boldsymbol{b}_{i}=(10+\epsilon, 0)$, there are no ties. The bidder always wins good 1 and wins/loses good 2 with .5 probability: $U^{w^{*}}((10+\epsilon, \epsilon))=.5[(15-10)]+.5[(-10)]=-2.5$.

- When $\boldsymbol{b}_{i}=(10-\epsilon, 0)$, there are no ties. The bidder always loses good 1 and wins/loses good 2 with .5 probability: $U^{w^{*}}((10-\epsilon, \epsilon))=.5[(0)]+.5[(0)]=0$. 
If $n$ is sufficiently large, $i$ 's optimal bid vector is thus $b_{i}^{*}=(10, \epsilon)$. However, under an approximate allocation rule that assumes a tie is among only two bidders, expected utility is $U^{\hat{w}_{p}}((10, \epsilon))=.5[.5(15-$ $10)+.5(0)]+.5[.5(-10)+.5(0)]=-1.25$. Theorem 16 is thus violated when $p=0.5$ and $\tau<1.25$.

Corollary 20. Theorem 18 does not necessarily hold if Assumption 7 is violated.

Proof. The same example given in the proof of Proposition 19 applies here. The optimal bid vector $\hat{\boldsymbol{b}}_{i}^{*}=$ $\max _{\boldsymbol{b}}\left\{U^{\hat{w}_{p}}(\boldsymbol{b})\right\}$ with respect to $\hat{w}_{p}$ is to not bid at all for expected utility 0 . However, $U_{i}^{w^{*}}\left(\boldsymbol{b}^{*}\right)$ approaches 1.25 as the number of players $n$ approaches $\infty$. Theorem 18 is thus violated when $n$ is sufficiently large and $0<\tau<1.25$.

\section{B Approximate Price Prediction}

We have shown (Lemma 1) that an optimal PP strategy is a best response to other-agent strategies, if price predictions exactly reflect the other-agent highest-bid distribution. Since it is unrealistic to expect perfect price prediction, we examine the consequences of employing PP strategies given imperfect price predictions.

We measure the distance between probability distributions in two ways. The first is a multivariate form of the Kolmogorov-Smirnov $(K S)$ statistic: $K S\left(\Pi, \Pi^{\prime}\right) \equiv \sup _{\boldsymbol{q}}\left|\Pi(\boldsymbol{q})-\Pi^{\prime}(\boldsymbol{q})\right|$. Second, we define the bundle probability distance $(B P)$ with respect to a bid $b$ :

$$
B P\left(\Pi, \Pi^{\prime}, \boldsymbol{b}\right)=\frac{1}{2} \sum_{X \subseteq \mathcal{X}}\left|\operatorname{Pr}_{\boldsymbol{q} \sim \Pi}(w(\boldsymbol{b}, \boldsymbol{q})=X)-\operatorname{Pr}_{\boldsymbol{q} \sim \Pi^{\prime}}(w(\boldsymbol{b}, \boldsymbol{q})=X)\right| .
$$

$K S$ is a generic measure of distance between two cumulative distributions. $B P$ measures the net movement of probability mass across regions defining the winning events for a given bid. The factor of $1 / 2$ corrects for the double-counting of probability mass in the origin and destination regions. Bundle probability distance directly captures the probability that the two distributions disagree about winnings for a given bid.

To analyze the impact of imperfect price predictions, we separately consider the effects on expected value of winnings and payment. Overall expected utility is the difference between these:

$$
\mathbb{E}_{\boldsymbol{q}}\left[u_{i}(\boldsymbol{b}, \boldsymbol{q})\right]=\mathbb{E}_{\boldsymbol{q}}\left[v_{i}(w(\boldsymbol{b}, \boldsymbol{q}))\right]-\mathbb{E}_{\boldsymbol{q}}[\psi(\boldsymbol{b}, \boldsymbol{q})] .
$$

We first consider payment. The following lemma bounds the effect on expected payment of imperfect price predictions.

Lemma 21. Let $\delta_{K S}=K S\left(\Pi, \Pi^{\prime}\right)$ and $\|\boldsymbol{b}\|_{1} \equiv \sum_{j=1}^{m} b^{j}$. Then for all $\boldsymbol{b}$,

$$
\mathbb{E}_{\boldsymbol{q} \sim \Pi}[\psi(\boldsymbol{b}, \boldsymbol{q})] \leq \mathbb{E}_{\boldsymbol{q} \sim \Pi^{\prime}}[\psi(\boldsymbol{b}, \boldsymbol{q})]+2 \delta_{K S}\|\boldsymbol{b}\|_{1},
$$

Proof. Since the total payment is the sum of the individual-good payments, the expected total payment is the sum of the expected individual-good payments. Let us therefore consider a particular good $j$. Further let us take $\Pi^{\prime}$ to be the baseline distribution, and $\Pi$ some variant on this baseline.

The variant $\Pi$ can increase expected payment for $j$ in two ways: in cases where the agent is winning $j$, by increasing prices such that $j$ is still won; or, in cases where the agent is not winning $j$, by decreasing prices so that $j$ is more likely to be won. The first effect is bounded by a situation where probability mass $\delta_{K S}$ is shifted from $p_{j}=0$ to $p_{j}=b^{j}-\epsilon$, for any $\epsilon>0$. This increases expected payments by no more than $\delta_{K S} b^{j}$; any greater shift would violate the constraint on KS distance. At the same time, we can shift another probability mass $\delta_{K S}$ from a point $p_{j}>b^{j}$, to $p_{j}=b^{j}-\epsilon$, which creates the second effect-it increases the probability of $b^{j}$ winning by $\delta_{K S}$, and thus expected payment by another at most $\delta_{K S} b^{j}$. Doing the same for every good yields a total expected payment increase bounded above by $2 \delta_{K S}\|\boldsymbol{b}\|_{1}$. 
This bound is tight. Consider a single good. Suppose the baseline prediction is $[(0.5,0) ;(0.5,20)]$ (i.e., with probability 0.5 , the price will be zero; otherwise it will be 20), and consider a bid of 10 . At the baseline, expected payment is zero: either the good is won at a price of zero, or the good is not won (again, at a cost of zero). Now change the probability distribution to $[(0.5-\delta, 0) ;(2 \delta, 10-\epsilon) ;(0.5-\delta, 20)]$, for some arbitrarily small $\epsilon>0$. Observe that this variant distribution satisfies the KS constraint: the new CDF differs from the old by $\delta:-\delta$ in the range $[0,10-\epsilon)$ and by $+\delta$ in the range $[10-\epsilon, 20)$. Further, a bid of 10 wins the good at a price of 10 (essentially) with probability $2 \delta$, so the expected payment is $20 \delta$.

We can similarly bound the effect of inaccurate price predictions on expected value of winnings. A variant distribution can degrade expected value of winnings only by decreasing the probability of winning valuable bundles. By constraining $B P$ distance, we ensure that, for any set of bundles, the total probability of winning a bundle from that set at bid vector $\boldsymbol{b}$ can decrease by at most $\delta_{B P}$. This means that the expected value of winnings can suffer by at most $\delta_{B P} \bar{V}$.

Lemma 22. Let $\delta_{B P}=B P\left(\Pi, \Pi^{\prime}, \boldsymbol{b}\right)$. Then

$$
\mathbb{E}_{\boldsymbol{q} \sim \Pi[}\left[v_{i}(w(\boldsymbol{b}, \boldsymbol{q}))\right] \geq \mathbb{E}_{\boldsymbol{q} \sim \Pi^{\prime}}\left[v_{i}(w(\boldsymbol{b}, \boldsymbol{q}))\right]-\delta_{B P} \bar{V}
$$

Combining the lemmas, we have the following bound on expected utility (46).

Theorem 23. Let $\delta_{K S}=K S\left(\Pi, \Pi^{\prime}\right)$ and $\delta_{B P}=B P\left(\Pi, \Pi^{\prime}, \boldsymbol{b}\right)$. Then for all $i$,

$$
\mathbb{E}_{\boldsymbol{q} \sim \Pi}\left[u_{i}(\boldsymbol{b}, \boldsymbol{q})\right] \geq \mathbb{E}_{\boldsymbol{q} \sim \Pi^{\prime}}\left[u_{i}(\boldsymbol{b}, \boldsymbol{q})\right]-\left(\delta_{B P} \bar{V}+2 \delta_{K S}\|\boldsymbol{b}\|_{1}\right) .
$$

Henceforth, let us denote by $\bar{b}$ the maximum payment, that is, the $L_{1}$-norm of the greatest possible bid vector. The value of $\bar{b}$ is bounded above by $m \bar{V}$ for any rational bidding strategy under any valuation distribution, but typically it will be far less than that.

Lemma 24. Let $U(s, \Pi)$ denote an agent's expected utility when playing strategy $s$ while the other-agent highest-bid distribution is $\Pi$. Further, suppose that strategy $\hat{s}^{*}$ is a best response to some $\hat{\Pi}$, while $\Pi$ is the highest other-agent bid distribution actually induced by $\hat{s}^{*}$, and that $s^{*}$ is a best response to $\Pi$. If $K S(\hat{\Pi}, \Pi) \leq \delta_{K S}$, and for all $\boldsymbol{b}, B P(\hat{\Pi}, \Pi, \boldsymbol{b}) \leq \delta_{B P}$, then $U\left(\hat{s}^{*}, \Pi\right) \geq U\left(s^{*}, \Pi\right)-2\left(\delta_{B P} \bar{V}+2 \delta_{K S} \bar{b}\right)$.

Proof.

$$
\begin{aligned}
U\left(\hat{s}^{*}, \Pi\right) & \geq U\left(\hat{s}^{*}, \hat{\Pi}\right)-\left(\delta_{B P} \bar{V}+2 \delta_{K S} \bar{b}\right) \\
& \geq U\left(s^{*}, \hat{\Pi}\right)-\left(\delta_{B P} \bar{V}+2 \delta_{K S} \bar{b}\right) \\
& \geq U\left(s^{*}, \Pi\right)-2\left(\delta_{B P} \bar{V}+2 \delta_{K S} \bar{b}\right)
\end{aligned}
$$

Inequality (49) follows from the constraints on distance and Theorem 23. The fact that $\hat{s}^{*}$ is a best response to $\hat{\Pi}$ yields (50), and another invocation of Theorem 23 produces (51).

Applying this lemma to each agent in turn yields the conditions for approximate equilibrium.

Theorem 25. Suppose that, for each agent $i$, strategy $\hat{s}_{i}^{*}$ is a best response to $\hat{\Pi}_{i}$, while $\Pi$ is the highest other-agent bid distribution actually induced by $\hat{s}_{-i}^{*}$. If for all agents $i, K S\left(\hat{\Pi}_{i}, \Pi\right) \leq \delta_{K S}$, and for all $\boldsymbol{b}$, $B P\left(\hat{\Pi}_{i}, \Pi, \boldsymbol{b}\right) \leq \delta_{B P}$, then $\hat{\boldsymbol{s}}^{*}$ constitutes an $\epsilon$-Bayes-Nash equilibrium, for $\epsilon=2 \delta_{B P} \bar{V}+4 \delta_{K S} \bar{b}$. 

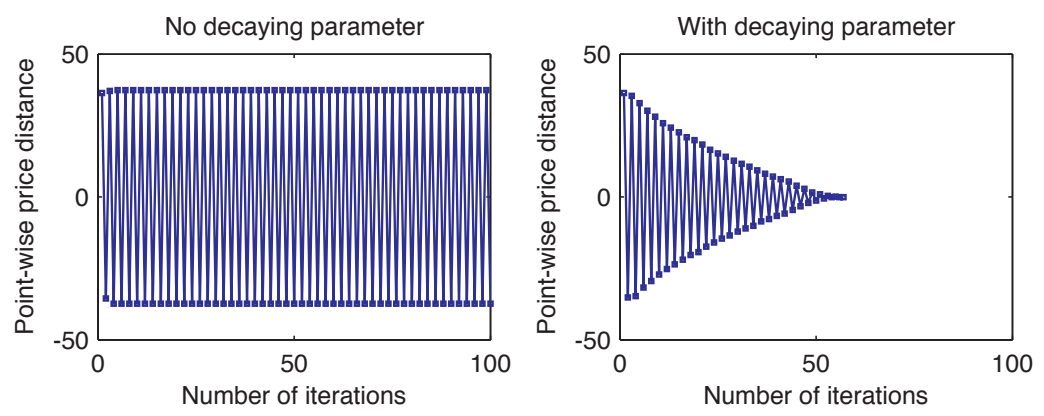

Figure 6: Effect of the decay factor on the iterative SCPP procedure, for PP strategy TargetMV*.

\section{Evaluation of Self-Confirming Price Prediction}

Here we present a detailed evaluation of the procedure for deriving self-confirming price predictions, introduced in $§ 5.6$. Our computational experiments employ the two valuation environments used for empirical game-theoretic analysis. In $U[m, n](\S 6.2 .1)$, there are $n$ agents with complementary and substitutable preferences over $m$ goods. In $H[m, n](\S 6.2 .2)$, the $n$ agents consider the $m$ goods to be perfect substitutes.

\section{C.1 Convergence}

We evaluated the convergence of this procedure using the parameter settings shown in Table $1\left(G=10^{6}\right.$ and $L=100$ ) with respect to three different point PP strategies: StraightMV, TargetMV, and TargetMV*. These runs adopt the price (not HB) convention for interpreting price outcomes. For StraightMV, we reach a fixed point within 20 iterations, even without damping $\left(\kappa_{t}=1\right)$. For TargetMV and TargetMV*, the price predictions tend to oscillate, hence we need a decay schedule to settle the process.

The left-hand plot of Figure 6 depicts a series of pointwise price distances between consecutive iterations in deriving point price predictions for TargetMV* with $\kappa_{t}=1$, for an environment featuring complements. Without damping, the iterative update of price predictions for TargetMV* fails to converge. Since TargetMV* bids for goods in its target bundle under the assumption that no others are available, these bids tend to be at the extremes of its value range. With all agents playing this strategy, this leads to high prices, which causes changes in targets in the next iteration (by all agents), causing these same goods to have very low prices then. ${ }^{11}$ With updates subject to decay sequence $\kappa_{t}=\frac{L-t+1}{L}$ (plot at right), the oscillations are effectively damped and the process converges to an approximately self-confirming price prediction.

Next, we examine the convergence pattern of the iterative procedure for distribution predictions. Figure 7 displays the $K S_{\text {marg }}$ statistic at each iteration for the derivation of SCPPs for three different strategies, using the HB outcome interpretation, in the $U[5,4]$ environment. As we can see, the process eventually reaches an approximate marginally self-confirming prediction for each strategy. The convergence is not smooth or even monotone, owing it seems to excessive oscillation in the early rounds of the update process. Oscillations remain even when the prediction approaches self-confirming, but the damped updates keep it within the accurate range. Alternative decay schedules $\kappa_{t}$ would likely lead to different convergence patterns.

\footnotetext{
${ }^{11}$ TargetMV produces a similar pattern, though attenuated by its consideration of alternative buying opportunities.
} 


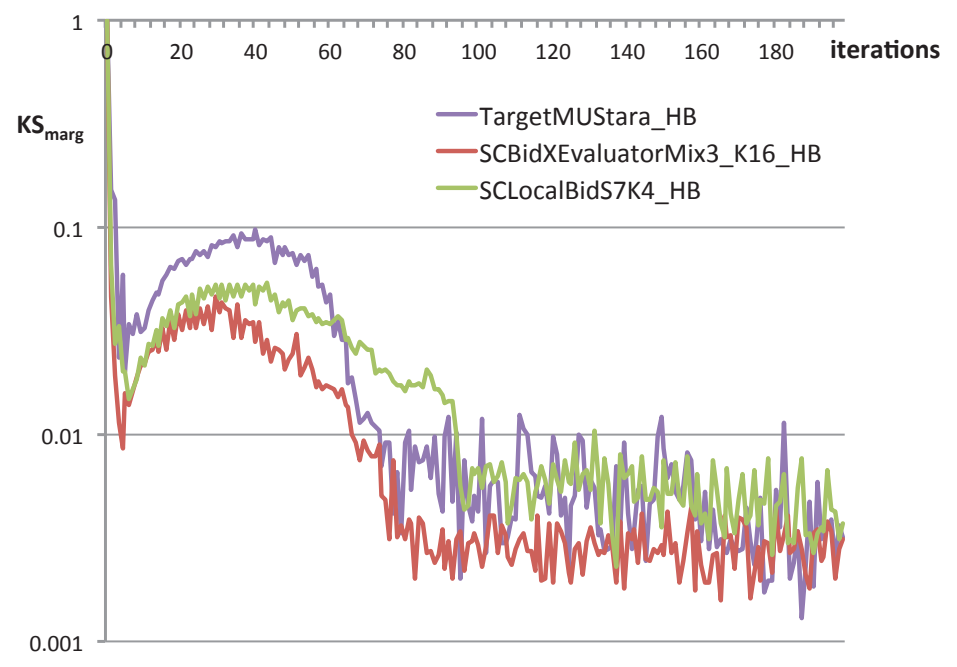

Figure 7: Accuracy of self-confirmation ( $K S_{\text {marg }}$, log scale) over the iterative update process, for three strategies in SimSPSB environment $U[5,4]$. The procedure employs parameter settings from Table 1, with $G=10^{5}$ and $L=200$.

\section{C.2 Accuracy}

The decay parameter ensures that the iterative procedure for deriving price predictions will terminate, but potentially masks violations of self-confirmation in the result. To test the accuracy of the self-confirming price predictions calculated for this study (both point and distribution), we ran an extra iteration (i.e., $G$ additional game instances) after a supposed SCPP was returned by the procedure. The distances between the supposed SCPPs and the output of an extra iteration, for a variety of PP strategies, are reported in Table 4. As shown in the table, all are self-confirming to a fine level of approximation. For point price predictions, the maximal error observed for any good is 0.066 price units. For distribution predictions, the maximal cumulative probability error for any good price is less than 0.01 .

\begin{tabular}{|r||r|}
\hline Point Strategy & $\Delta$ Statistic \\
\hline StraightMV & 0.0116 \\
TargetMV $^{*}$ & 0.0660 \\
TargetMV $^{*}$ & 0.0553 \\
\hline \hline Distribution Strategy & $K S_{\text {marg }}$ Statistic \\
\hline StraightMU64 & 0.00450 \\
TargetMU64 & 0.00879 \\
TargetMU*64 & 0.00788 \\
AverageMU64 & 0.00516 \\
\hline
\end{tabular}

Table 4: Accuracy of self-confirming point and distribution price predictions.

Finally, we evaluate the accuracy of price predictions with respect to the full joint distribution. Since our search procedure seeks only marginal self-confirmation, we should not expect a high degree of accuracy once interdependencies are taken into account. Nevertheless, relative accuracy may provide an indication of the usefulness of the procedure. Our evaluation considers the environments $U[5,5]$ and $H[5,5]$. For each PP strategy and prediction combination evaluated, we estimated the $K S$ statistic by randomly generating 4000 
price vectors and evaluating the empirical cumulative probability at each of these points, over $10^{6}$ simulated self-play game instances.

Prediction accuracy results are shown in Figure 8. The strategies are described in Appendix E, but for current purposes the name suffices to convey the three features of interest: (1) strategy class (AverageMU, StraightMU, BidEval, or LocalBid), (2) whether the prediction was derived using the HB (name ends in $\mathrm{HB}$ ) or price outcome interpretation, and (3) whether the prediction was derived to be self-confirming for this strategy (AverageMU, or name beginning with SC). We note first that the $K S$ values are significantly above zero, so even if the strategies were found to be marginally self-confirming, the cumulative joint distributions exhibit substantial error at least at some points. Next, we observe that for instances differing only on the outcome interpretation (HB or price), the HB prediction is more accurate in four out of five cases - and the fifth is quite close. This is as expected, given that HB is the relevant prediction target. Finally, in all eight instances where strategies differ only on whether self-confirming prices are employed, the SC prediction is more accurate, often dramatically so. This last observation suggests that marginally self-confirming prices behave qualitatively in line with actual self-confirming distributions, even though the independence implicitly assumed does not approximately hold.

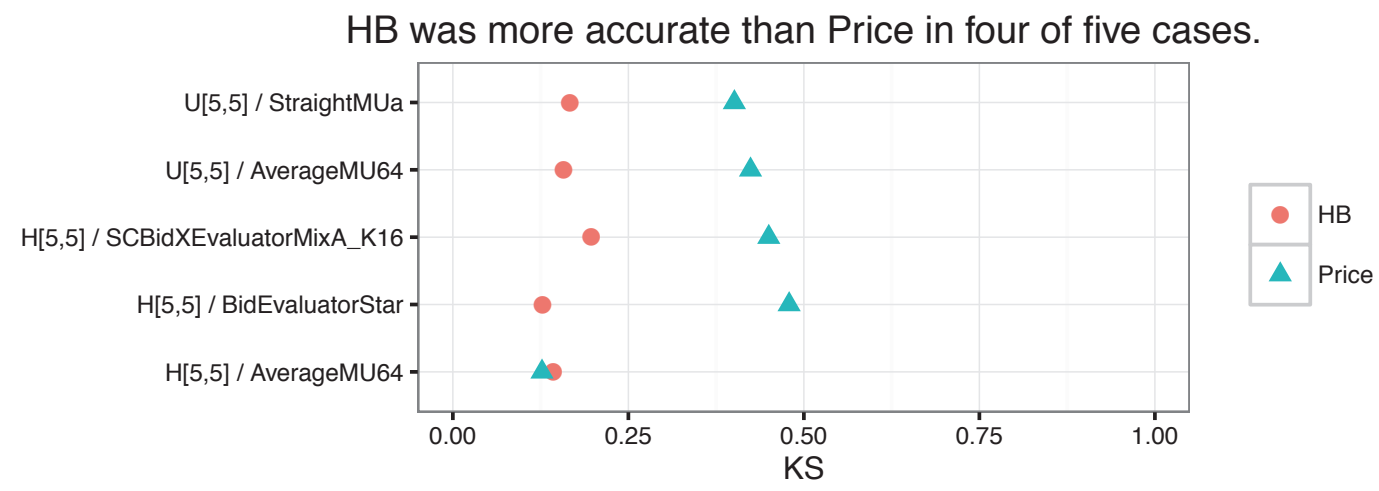

SC was more accurate than Non-SC in all cases.

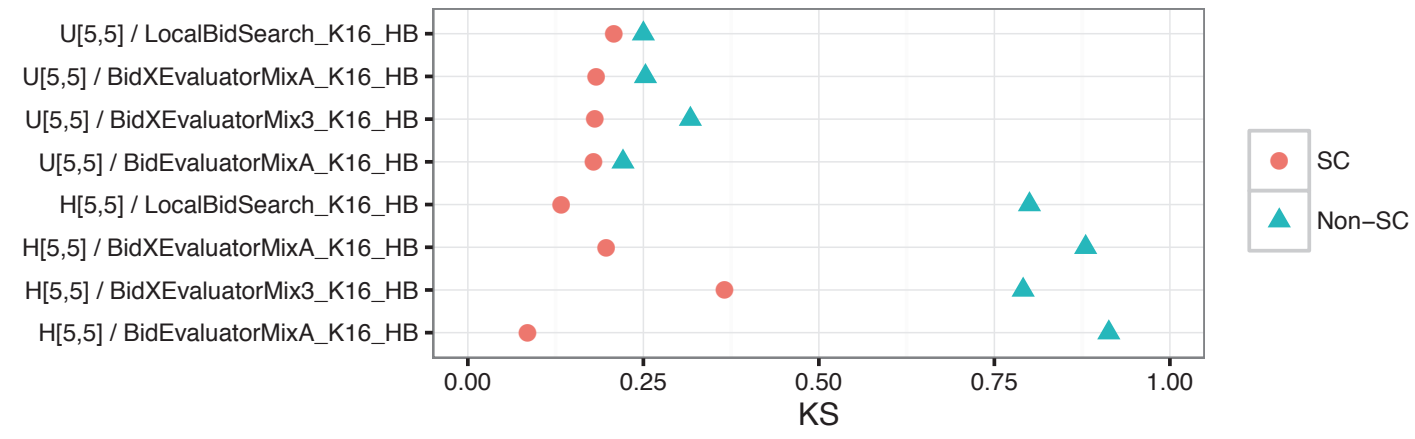

Figure 8: Comparative joint price prediction accuracy when employing HB (top) and SC (bottom) versions of a given strategy. 


\section{Bid Optimization Performance}

To relate the strategic and decision-theoretic performance of our heuristic bidding strategies, we performed a systematic assessment of their effectiveness in optimizing bids, assuming correct price predictions. We additionally analyze how and when the various bidding strategies succeed and fail, with respect to the instances of a valuation distribution with complementarities.

\section{D.1 Evaluation Framework}

We conduct our evaluation in SimSPSB environment $U[5,5]$. We evaluate three price-prediction strategies: AverageMU64_HB, LocalBidSearch_K16_HB, and BidXEvaluatorMix3_K16_HB (subsequently termed AverageMU, LocalBid, and BidEval, respectively). As input, all bidding strategies were given self-confirming prices generated by AverageMU, shown in Figure 9(a). For each strategy, we sampled 5000 valuations and generated bids according to that strategy. We also approximated an optimal bid vector for each sampled valuation, using the OPT heuristic (§5.4.3).

Expected utility (profit) for each bidding heuristic was compared to that produced by OPT. Given price prediction $\Pi$, we can express expected profit as the difference between expected winnings value and expected payment:

$$
\mathbb{E}_{\boldsymbol{q} \sim \Pi}\left[u_{i}(\boldsymbol{b}, \boldsymbol{q})\right]=\mathbb{E}_{\boldsymbol{q} \sim \Pi}\left[v_{i}(w(\boldsymbol{b}, \boldsymbol{q}))\right]-\mathbb{E}_{\boldsymbol{q} \sim \Pi} \sum_{j \in w(\boldsymbol{b}, \boldsymbol{q})} q_{j}
$$

Under our assumption that the distributions over each good are independent, $\Pi(q)=\prod_{j=1}^{m} \Pi_{j}\left(q_{j}\right)$, expected payment is

$$
\mathbb{E}_{\boldsymbol{q} \sim \Pi} \sum_{j \in w(\boldsymbol{b}, \boldsymbol{q})} q_{j}=\sum_{j=1}^{m} \int_{q=0}^{b^{j}} q d \Pi_{j}(q) .
$$

For scheduling valuations $(\S 6.2 .1)$, expected value of winnings is

$$
\mathbb{E}_{\boldsymbol{q} \sim \Pi}\left[v_{i}(w(\boldsymbol{b}, \boldsymbol{q}))\right]=\sum_{j=1}^{m} \gamma_{j-1}^{\lambda-1}(\boldsymbol{b}) \Pi_{j}\left(b^{j}\right) v^{j},
$$

where $\gamma_{k}^{l}(\boldsymbol{b})$ denotes the probability that exactly $l$ out of the first $k$ goods are obtained. This can be expressed recursively:

$$
\gamma_{k}^{l}(\boldsymbol{b})=\left\{\begin{aligned}
\gamma_{k-1}^{l-1}(\boldsymbol{b}) \Pi_{k}\left(b^{k}\right)+\gamma_{k-1}^{l}(\boldsymbol{b})\left(1-\Pi_{j}\left(b^{k}\right)\right) & \text { if } k \geq \max (l, 1) \\
1 & \text { if } k=l=0 \\
0 & \text { otherwise. }
\end{aligned}\right.
$$

Since both LocalBid and AverageMU can produce bids with negative expected profit, we also consider two simple extended strategies, LocalBid-post and AverageMU-post, which perform a post-processing step of calculating expected profit for the proposed bid vector, and if that is negative return a null bid.

\section{D.2 OPT Bids}

Figures 9(b) and 9(c) present information about the bids computed by OPT for the given price predictions. As seen in Figure 9(b), profitability decreases with higher $\lambda$ values, and not bidding at all is frequently optimal when $\lambda=5$. Intermediate $\lambda$ values likely pose the greatest challenge for bidding heuristics, since the instances maintain a high degree of complementarity, yet there is more potential profit to lose by bidding suboptimally. 


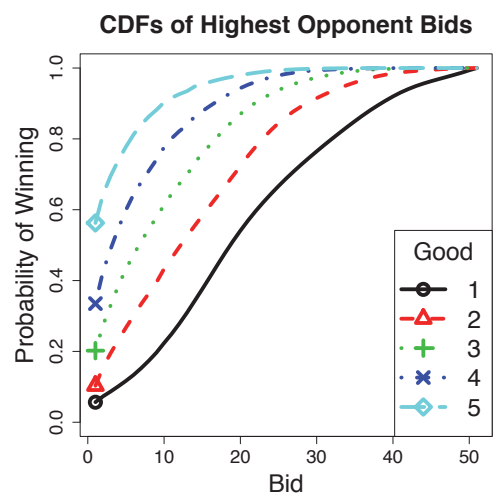

(a)

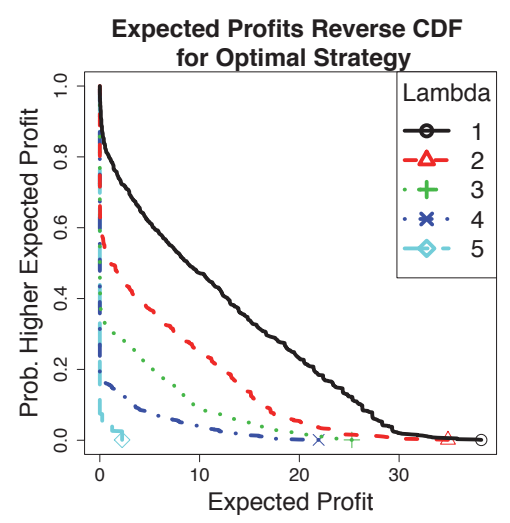

(b)

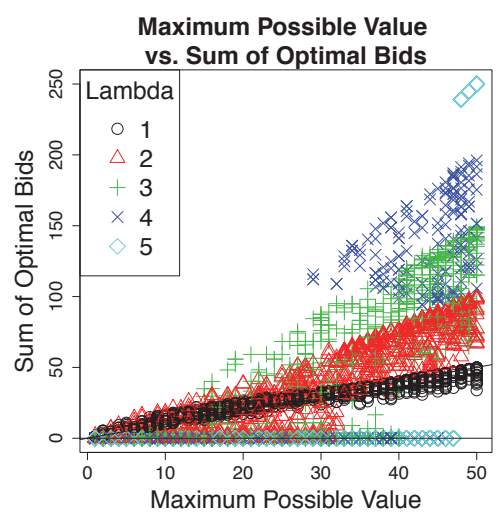

(c)

Figure 9: (a) Cumulative distribution function over other-agent bids, based on the self-confirming prices generated by AverageMU. (b) Reverse cumulative distribution function over expected profit for OPT. (c) Maximum possible winnings value versus worst-case amount paid by OPT bids.

Figure 9(c) shows the maximum value an agent could receive for a given instance (i.e., the value for winning the earliest $\lambda$ items) versus the sum of the OPT bids for that instance. It is often most profitable to bid in aggregate about $\lambda$ times higher than the maximum value. The high aggregate bids with respect to value demonstrate that OPT bidders are willing to expose themselves to the risk of paying high prices in order to avoid the chance of winning goods but still failing to meet their $\lambda$ threshold.

\section{D.3 Evaluation Results}

Table 5 compares the expected profit of each strategy to OPT. The variation of OPT expected profit across strategies is due to variation in sampling the 5000 valuations; these differences were not statistically significant $(p=.452) .{ }^{12}$ Each strategy similarly has a high standard deviation in expected profit, although the differences between strategies in terms of absolute distance from OPT were found to be statistically significant in all cases $(p<.0001)$ except for the difference between LocalBid and LocalBid-post $(p=.970) .{ }^{13}$

\begin{tabular}{|r||rr||rr|rr|r|}
\hline \multicolumn{1}{|c||}{ Strategy } & \multicolumn{2}{c||}{ OPT profit } & \multicolumn{2}{|c|}{ ALG profit } & \multicolumn{2}{c|}{ (OPT-ALG) profit } & \multirow{2}{*}{ \% OPT } \\
& mean & s.d. & mean & s.d. & mean & s.d. & \\
\hline LocalBid-post & 3.94 & $(7.15)$ & 3.90 & $(7.16)$ & 0.05 & $(0.35)$ & 98.77 \\
LocalBid & 3.94 & $(7.15)$ & 3.89 & $(7.16)$ & 0.05 & $(0.35)$ & 98.67 \\
BidEval & 4.01 & $(7.18)$ & 3.79 & $(7.13)$ & 0.22 & $(0.61)$ & 94.49 \\
AverageMU-post & 4.34 & $(7.52)$ & 3.73 & $(7.13)$ & 0.61 & $(1.25)$ & 85.91 \\
AverageMU & 4.34 & $(7.52)$ & 2.86 & $(7.71)$ & 1.47 & $(2.53)$ & 66.10 \\
\hline
\end{tabular}

Table 5: Performance of each bidding strategy (ALG) with respect to OPT.

Next, we examine the performance of each bidding heuristic in more detail: Figure 10 shows each strategy's expected profit compared to that of OPT, for every sampled valuation instance.

\footnotetext{
${ }^{12}$ Checking for a significant difference in at least one of the groups was done using Kruskal-Wallis Rank Sum Test.

${ }^{13}$ All $p$-values in these comparisons were calculated using Pairwise Wilcoxon Rank Sum Tests, adjusting for multiple measures using the Bonferroni correction.
} 


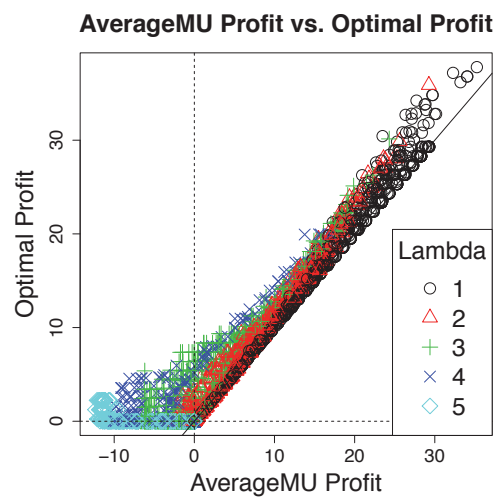

(a)

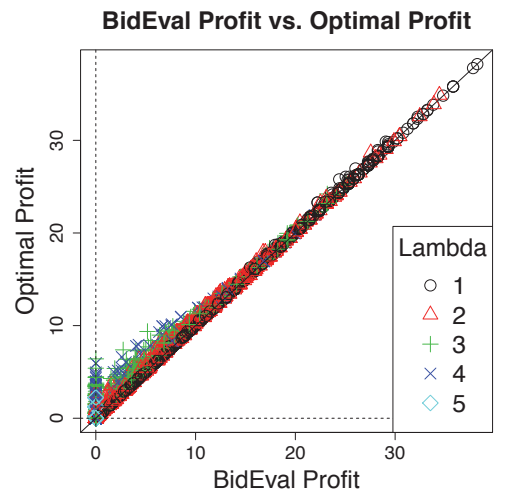

(b)

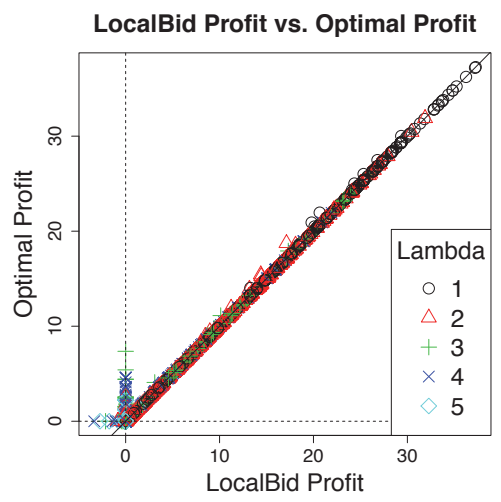

(c)

Figure 10: Expected profit for heuristic bidding strategies versus OPT, for each of 5000 valuations. Points above the diagonal represent suboptimal instances for the strategy.

AverageMU Figure 10(a) shows that AverageMU frequently places bids with negative expected profit (51.62\% of all instances). The majority of these negative expected profit instances were for higher $\lambda$ values, meaning greater complementarity and risk of exposure (see Table 6). As shown in Table 5, by eliminating negative expected profit instances, AverageMU-post improves performance to nearly $86 \%$ of OPT.

\begin{tabular}{|r||r|rrrrr|}
\hline Strategy & Negative & \multicolumn{5}{|c|}{ Fraction of Negatives with $\lambda$ Value } \\
& Instances & 1 & 2 & 3 & 4 & 5 \\
\hline AverageMU & 2581 & 0 & 0.1259 & 0.2534 & 0.3034 & 0.3173 \\
LocalBid & 17 & 0 & 0.1765 & 0.2353 & 0.4118 & 0.1765 \\
\hline
\end{tabular}

Table 6: Distribution across $\lambda$ values for bids with negative expected profit.

BidEval BidEval improves over AverageMU in terms of expected profit, but there are some valuation instances for which no bids are placed by BidEval, when some nonzero bid would have been profitable. The potential success or failure of BidEval lies in its bid generation strategy. Because it cannot explore the space of candidate bid vectors exhaustively, a tradeoff exists between diversifying, so that the space of bid vectors is suitably explored, and focusing on candidate bid vectors that other reasonably-profitable heuristics suggest. The instances in which no bids were placed are cases where every bid vector generated by BidEval's inner strategy had nonpositive expected profit.

BidEval performs proportionally worse on instances with lower optimal expected profit; this is likely due to the distribution of expected profits across $\lambda$ values. As seen in Figure 9(b), lower optimal expected profits occur more frequently for instances with higher $\lambda$ values, and the greater complementarity in these problems makes finding profitable bids more difficult.

LocalBid The main source of suboptimality for LocalBid is the vertical region at $x=0$ in Figure 10(c). This region represents lost opportunities-valuation instances where it would have been profitable to bid, but LocalBid did not do so. On inspection, we find that in most cases, OPT placed bids on the first $\lambda$ goods at points with high win probabilities. Since LocalBid considers bid changes for only one good at a time, any initialized bids that left LocalBid without enough certainty that it would receive at least $\lambda-1$ items resulted in LocalBid gradually opting out of bidding: if winning $\lambda$ goods were still unlikely after a bid change, the greedy improvement would not bid at all for that item. 

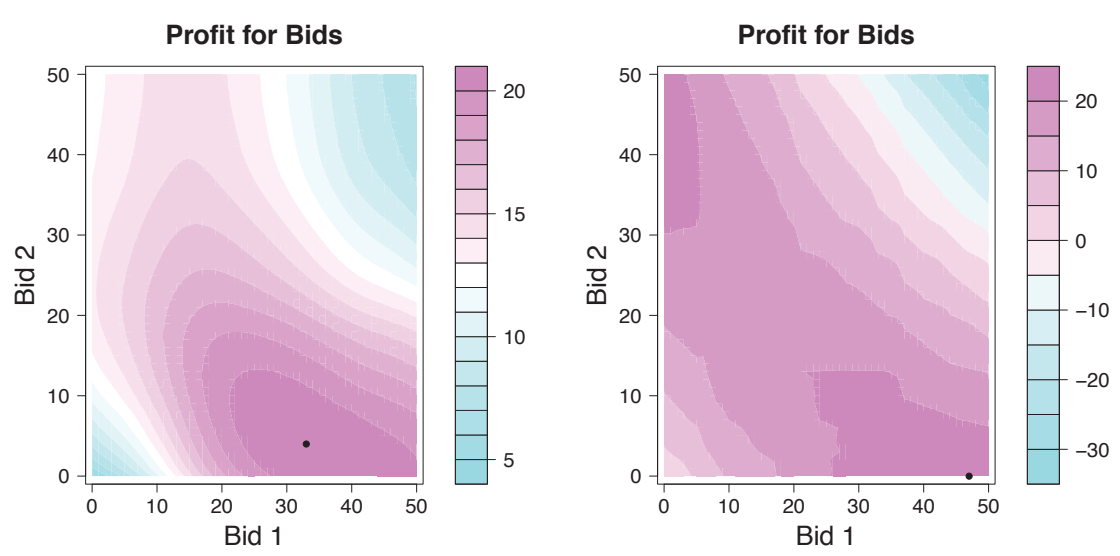

Figure 11: Expected profit visualizations for a two-good version of the scheduling environment.

The horizontal region at $y=0$ in Figure 10(c) shows that LocalBid also placed bids with negative expected profit, though it did so in less than $1 \%$ of all instances. Since this version of LocalBid uses AverageMU for its initial bid, we see that the local search escapes the negative profit region in most cases. In the cases where it did not, a local maximum was reached because the initialized bid vector satisfied the agent's $\lambda$ threshold with negative expected profit, and the reduced costs for not bidding on a single good were outweighed by the loss in value for no longer winning $\lambda$ items.

Overall, LocalBid optimizes quite well: $95.98 \%$ of all the sampled instances resulted in expected profits within $\$ 0.01$ of the OPT bid. It is somewhat surprising that a simple local search is so successful, but a visualization of the expected profit function provides some explanation. Figure 11 shows every possible bid vector's expected profit for a two-good version of the scheduling environment. Each plot is for some particular valuations and price predictions. For two goods, we see that the expected profit surface is quite smooth, causing LocalBid to converge to a unique global maximum regardless of initial bids. Numerous domain features could explain the smoothness of the expected profit function, such as (1) the particular values of self-confirming price predictions, (2) the price predictions being independent across goods, (3) the payment scheme of the second-price auction, or (4) the structure of scheduling valuations. Better understanding how these factors affect the quality of LocalBid's search, particularly when there are more than two goods, is left for future investigation. We note that, as shown in Figure 11, there do exist instances with multiple local maxima, even in the two-good environment.

\section{E Description of Strategies}

\section{E.1 A Non-Predictive Baseline Heuristic}

To calibrate the performance of the PP bidding strategies described in $\S 5$ (i.e., those based on price prediction), we include a baseline bidding strategy that employs no price information. Without price guidance, there is little basis to choose among alternative bundles. We therefore simply assume the agent selects one of its most valued sets of goods, which is an optimal acquisition at zero prices. Let $X^{*} \in \mathrm{ACQ}_{i}(\mathbf{0})$.

The BaselineBidding strategy bids for goods in $X^{*}$, assuming a total budget of $v\left(X^{*}\right)$. To determine the bid price for each individual good, it starts with the value for each good taken individually, and evenly 
divides the remaining budget among the goods. More precisely, let the excess value be given by

$$
e=v\left(X^{*}\right)-\sum_{x \in X^{*}} v(\{x\}) .
$$

BaselineBidding then bids for good $j$ as follows:

$$
\text { BaselineBidding }_{j}= \begin{cases}\max \left\{0, v(\{j\})+\frac{e}{\left|X^{*}\right|}\right\} & \text { if } j \in X^{*} \\ 0 & \text { otherwise. }\end{cases}
$$

\section{E.2 Table of Strategies}

Table 7 enumerates the strategies employed in our computational experiments, and presents their defining features and parameters. For the LocalBid strategies, the column "BE samples" is interpreted as the number of iterations through the goods, and "BE candidates" specifies the number of restarts.

Several BidEval strategies use a mix of heuristic methods for candidate bid generation. The mix is implemented by cycling through a fixed sequence of methods, until the quota of candidates is reached. Our strategies employ three different mix sequences:

mix1 StraightMU, TargetMU*

mix2 StraightMU, TargetMU*, AverageMU

mix3 StraightMU, TargetMU*, AverageMU128

Some additional features are inherent in the strategy name. Strategies ending in HB employ the highest other-agent bid for purposes of deriving self-confirming predictions. Those without HB employ the actual good price. BidEval strategies starting with BidX, as well as expected-value method ending in MUa or MUStara, employ exact evaluation of bids on a piecewise version of the price prediction, as opposed to sampling.

Distribution price predictions are represented as histograms, discretized at the level of integer prices. By default, strategies interpret bin $k, k \in\{0, \ldots, \bar{V}-1\}$, as the probability that the price lies in the interval $[k, k+1)$. Strategies with a $\mathrm{Z}$ in their name use bins $k \in\{0, \ldots, \bar{V}\}$ to represent the probability that the price lies in the interval $(k-1, k]$. Under either interpretation, strategies that sample from the prediction distribution draw a bin according to the histogram and return the integer at the lower bound of that bin. The sole exception is AverageMU64Zi_HB, which interpolates fractional prices when sampling.

\section{Acknowledgments}

This research was supported in part by Grants CCF-0905139 and IIS-1421391 from the U.S. National Science Foundation. Victor Naroditskiy and Jiacui Li provided useful feedback on the theoretical results. Dong Young Yoon developed the simulator and initiated the empirical game analysis. Brandon Mayer offered useful insight on the derivation of self-confirming predictions. Ben Cassell designed and implemented the testbed facility used to conduct our extensive simulations, and Boyang Zhu performed the bootstrap analysis. Anonymous reviewers provided numerous constructive suggestions to improve the work. 


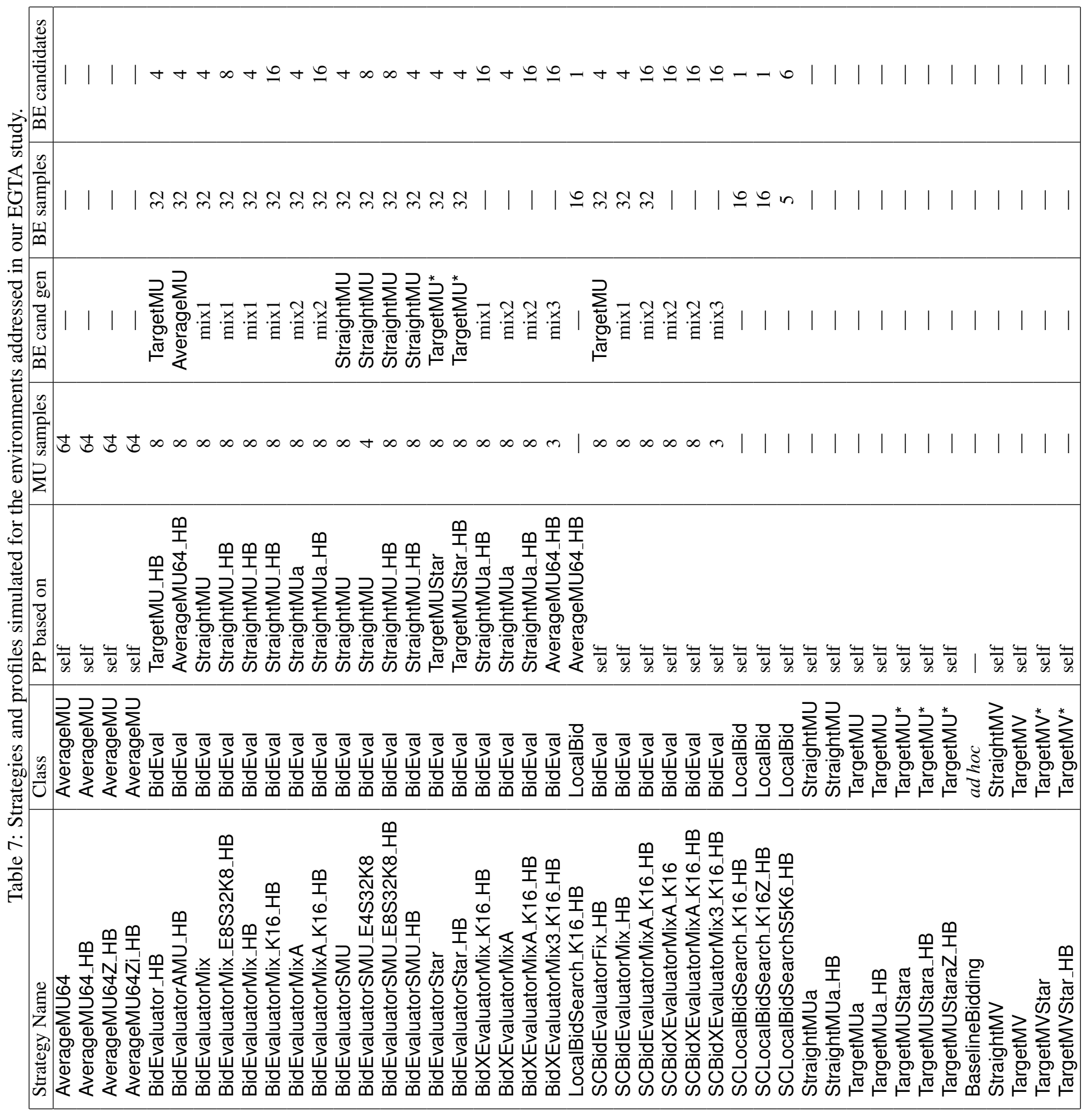




\section{References}

John Birge and Francois Louveaux. Introduction to Stochastic Programming. Springer, 1997.

Justin Boyan and Amy Greenwald. Bid determination in simultaneous auctions: An agent architecture. In Third ACM Conference on Electronic Commerce, pages 210-212, Tampa, 2001.

Yang Cai and Christos Papadimitriou. Simultaneous Bayesian auctions and computational complexity. In Fifteenth ACM Conference on Economics and Computation, pages 895-910, 2014.

Ben-Alexander Cassell and Michael P. Wellman. EGTAOnline: An experiment manager for simulationbased game studies. In Multi-Agent Based Simulation XIII, volume 7838 of Lecture Notes in Artificial Intelligence. Springer, 2013.

George Christodoulou, Annamária Kovács, and Michael Schapira. Bayesian combinatorial auctions. In 35th International Colloquium on Automata, Languages and Programming, pages 820-832, Reykjavik, 2008.

Peter Cramton. Simultaneous ascending auctions. In Cramton et al. [2005].

Peter Cramton, Yoav Shoham, and Richard Steinberg, editors. Combinatorial Auctions. MIT Press, 2005.

Richard Engelbrecht-Wiggans and Robert J. Weber. An example of a multi-object auction game. Management Science, 25:1272-1277, 1979.

Michal Feldman, Hu Fu, Nick Gravin, and Brendan Lucier. Simultaneous auctions are (almost) efficient. In 45th Annual ACM Symposium on Theory of Computing, pages 201-210, 2013.

Herbert Gintis. Game Theory Evolving. Princeton University Press, 2000.

Amy Greenwald and Justin Boyan. Bidding under uncertainty: Theory and experiments. In Twentieth Conference on Uncertainty in Artificial Intelligence, pages 209-216, Banff, 2004.

Amy Greenwald, Seong Jae Lee, and Victor Naroditskiy. RoxyBot-06: Stochastic prediction and optimization in TAC travel. Journal of Artificial Intelligence Research, 36:513-546, 2009.

Amy Greenwald, Victor Naroditskiy, and Seong Jae Lee. Bidding heuristics for simultaneous auctions: Lessons from TAC travel. In Wolfgang Ketter, Han La Poutré, Norman Sadeh, Onn Shehory, and William Walsh, editors, Agent-Mediated Electronic Commerce and Trading Agent Design and Analysis, volume 44 of Lecture Notes in Business Information Processing, pages 131-146. Springer-Verlag, 2010.

Amy Greenwald, Jiacui Li, and Eric Sodomka. Approximating equilibria in sequential auctions with incomplete information and multi-unit demand. In Advances in Neural Information Processing Systems, Lake Tahoe, 2012.

Avinatan Hassidim, Haim Kaplan, Yishay Mansour, and Noam Nisan. Non-price equilibria in markets of discrete goods (extended abstract). In Twelfth ACM Conference on Electronic Commerce, page 295, 2011.

Patrick R. Jordan. Practical Strategic Reasoning with Applications in Market Games. PhD thesis, University of Michigan, 2010. 
Patrick R. Jordan, Christopher Kiekintveld, and Michael P. Wellman. Empirical game-theoretic analysis of the TAC supply chain game. In Sixth International Joint Conference on Autonomous Agents and MultiAgent Systems, pages 1188-1195, Honolulu, 2007.

Paul Klemperer. Auctions: Theory and Practice. Princeton University Press, 2004.

Vijay Krishna. Auction Theory. Academic Press, second edition, 2010.

Vijay Krishna and Robert W. Rosenthal. Simultaneous auctions with synergies. Games and Economic Behavior, 17:1-31, 1996.

Jeffrey K. MacKie-Mason, Anna Osepayshvili, Daniel M. Reeves, and Michael P. Wellman. Price prediction strategies for market-based scheduling. In Fourteenth International Conference on Automated Planning and Scheduling, pages 244-252, Whistler, BC, 2004.

Brandon A. Mayer, Eric Sodomka, Amy Greenwald, and Michael P. Wellman. Accounting for price dependencies in simultaneous sealed-bid auctions. In Fourteenth ACM Conference on Electronic Commerce, pages 679-696, 2013.

Paul Milgrom. Putting auction theory to work: The simultaneous ascending auction. Journal of Political Economy, 108:245-272, 2000.

Victor Naroditskiy. Select Problems at the Intersection of Computer Science and Economics. PhD thesis, Brown University, August 2009.

Renato Paes Leme, Vasilis Syrgkanis, and Éva Tardos. The dining bidder problem: á la russe et á la française. SIGecom Exchanges, 11(2):25-28, 2012.

Zinovi Rabinovich, Victor Naroditskiy, Enrico H. Gerding, and Nicholas R. Jennings. Computing pure Bayesian-Nash equilibria in games with finite actions and continuous types. Artificial Intelligence, 195: 106-139, 2013.

Daniel M. Reeves, Michael P. Wellman, Jeffrey K. MacKie-Mason, and Anna Osepayshvili. Exploring bidding strategies for market-based scheduling. Decision Support Systems, 39:67-85, 2005.

Robert W. Rosenthal and Ruqu Wang. Simultaneous auctions with synergies and common values. Games and Economic Behavior, 17:32-55, 1996.

Peter Stone, Robert E. Schapire, Michael L. Littman, János A. Csirik, and David McAllester. Decisiontheoretic bidding based on learned density models in simultaneous, interacting auctions. Journal of Artificial Intelligence Research, 19:209-242, 2003.

Vasilis Syrgkanis. Bayesian games and the smoothness framework. Technical report, Cornell University, 2012.

Balázs Szentes and Robert W. Rosenthal. Three-object two-bidder simultaneous auctions: Chopsticks and tetrahedra. Games and Economic Behavior, 44:114-143, 2003.

Michael P. Wellman. Methods for empirical game-theoretic analysis (extended abstract). In Twenty-First National Conference on Artificial Intelligence, pages 1552-1555, Boston, 2006. 
Michael P. Wellman. Trading Agents. Morgan and Claypool, 2011.

Michael P. Wellman, Daniel M. Reeves, Kevin M. Lochner, and Yevgeniy Vorobeychik. Price prediction in a trading agent competition. Journal of Artificial Intelligence Research, 21:19-36, 2004.

Michael P. Wellman, Amy Greenwald, and Peter Stone. Autonomous Bidding Agents: Strategies and Lessons from the Trading Agent Competition. MIT Press, 2007.

Michael P. Wellman, Anna Osepayshvili, Jeffrey K. MacKie-Mason, and Daniel M. Reeves. Bidding strategies for simultaneous ascending auctions. B. E. Journal of Theoretical Economics (Topics), 8(1), 2008.

Michael P. Wellman, Lu Hong, and Scott E. Page. The structure of signals: Causal interdependence models for games of incomplete information. In Twenty-Seventh Conference on Uncertainty in Artificial Intelligence, pages 727-735, Barcelona, 2011.

Bryce Wiedenbeck, Ben-Alexander Cassell, and Michael P. Wellman. Bootstrap techniques for empirical games. In Thirteenth International Conference on Autonomous Agents and Multi-Agent Systems, pages 597-604, 2014.

Dong Young Yoon and Michael P. Wellman. Self-confirming price prediction for bidding in simultaneous second-price sealed-bid auctions. In IJCAI-11 Workshop on Trading Agent Design and Analysis, Barcelona, 2011. 